\title{
CONTINUOUS BIORTHOGONALITY OF AN ELLIPTIC HYPERGEOMETRIC FUNCTION
}

\author{
V. P. SPIRIDONOV
}

\begin{abstract}
A family of continuous biorthogonal functions related to an elliptic ana$\log$ of the Gauss hypergeometric function is constructed. The key tools used for that are the elliptic beta integral and the integral Bailey chain introduced earlier by the author. The relationship with the Sklyanin algebra and elliptic analogs of the Faddeev modular double are discussed in detail.
\end{abstract}

\section{$\S 1$. INTRODUCTION}

Classical and quantum completely integrable systems serve as a rich source of special functions. The complexity of these systems correlates with the complexity of solutions of the Yang-Baxter equation - rational, trigonometric, or elliptic 22. In the theory of special functions this structural hierarchy is reflected in the existence of the plain and $q$-hypergeometric functions [1] and their elliptic generalizations [19]. One of our aims in the present paper is to clarify certain questions about this correspondence at the elliptic level.

In 17, 19, the author introduced an elliptic analog of the Gauss hypergeometric function $V\left(t_{1}, \ldots, t_{8}\right)$ that generalizes many special functions of hypergeometric type obeying "classical" properties [1. In particular, it was shown that this function exhibits symmetry transformations tied to the exceptional root system $E_{7}$ and satisfies the elliptic hypergeometric equation. We start this work with showing in $\S 2$ that the $V$-function satisfies a simple biorthogonality relation corresponding to the continuous values of a spectral parameter. For that we use the elliptic beta integral [16] and an integral analog of the Bailey chains introduced in [18. Our construction can be viewed as an integral generalization of Rosengren's approach to the elliptic $6 j$-symbols [11.

In $\S 3$, we investigate the generalized eigenvalue problem for a second order finite difference operator $D(a, b, c, d ; p ; q)$. The relationship of such problems with biorthogonality relations was considered in 23. A problem similar to ours was investigated earlier in [10, 11 for the discrete spectrum. Its general solution determines a basis in some space of meromorphic functions. For a simple scalar product, we derive the dual basis defined by solutions of a similar spectral problem for conjugate operators. As a result, the overlap of the basis vectors with their duals coincides with the $V$-function. In $\S 4$, we derive a new contiguous relation for the elliptic hypergeometric function and the biorthogonality condition associated with that.

It turns out that the $V$-function is directly related to the Sklyanin algebra, one of the central objects in the quantum inverse scattering method [14, 15. This relationship is established in $\S 5$ on the basis of the observation, due to Rains 19, 12, that the $D$-operator can be represented as a linear combination of all four generators of the Sklyanin algebra.

2000 Mathematics Subject Classification. Primary 33C75, 81R12.

Key words and phrases. Completely integrable systems, special functions, Sklyanin algebra, Faddeev modular double. 
In this way we obtain a particular infinite-dimensional module of this algebra with the continuous values of the Casimir operators.

It is well known that the Sklyanin algebra can be degenerated to the quantum algebra $U_{q}\left(s l_{2}\right)$, which has been investigated intensively during the last two decades. In [3], Faddeev introduced the modular double - the quantum algebra $U_{q}\left(s l_{2}\right) \otimes U_{\tilde{q}^{-1}}\left(s l_{2}\right)$, where $\tilde{q}$ is a modular transform of $q$ (see also [6]). Originally, this construction was aimed at dealing with the well-defined logarithms of quantum algebra generators, which can be traced to the demand of the analytical uniqueness of the algebra representation modules. Applying the latter requirement to the results of $\S 5$, we come in $\S 6$ to two different elliptic analogs of the modular double defined by direct products of two copies of the Sklyanin algebra with generators related to each other by a permutation of certain parameters. An interesting fact is that some of the generators of these two algebras anticommute with each other.

Finally, in $\S 7$ we discuss the Bethe Anzatz for the D-operator eigenfunctions and relations to the Calogero-Sutherland type models - common topics in the theory of integrable systems. We demonstrate that the standard eigenvalue problem for the $D$-operator can be interpreted as a difference analog of the Heun equation. The latter equation is known to emerge in the one particle sector of the Inozemtsev integrable model [5]. Our finite difference analog of it turns out to be related to the model introduced by van Diejen 2. for a restricted set of parameters.

Before passing to the derivation of these results, we describe our notation. For an Abelian variable $z \in \mathbb{C}$ and a base $p=e^{2 \pi i \tau}, \operatorname{Im}(\tau)>0$ (i.e., $|p|<1$ ), we define the infinite product

$$
(z ; p)_{\infty}:=\prod_{j=0}^{\infty}\left(1-z p^{j}\right) .
$$

The elliptic theta functions with characteristics are defined by the series

$$
\theta_{a b}(u)=\sum_{k \in \mathbb{Z}} e^{\pi i \tau(k+a / 2)^{2}} e^{2 \pi i(k+a / 2)(u+b / 2)},
$$

where the variables $a$ and $b$ take values 0 or 1 , and $u \in \mathbb{C}$. The standard Jacobi theta functions are defined as

$$
\begin{aligned}
& \theta_{1}(u \mid \tau)=\theta_{1}(u)=-\theta_{11}(u) \\
& \theta_{2}(u \mid \tau)=\theta_{2}(u)=\theta_{10}(u)=\theta_{1}(u+1 / 2), \\
& \theta_{3}(u \mid \tau)=\theta_{3}(u)=\theta_{00}(u)=e^{\pi i \tau / 4+\pi i u} \theta_{1}(u+1 / 2+\tau / 2), \\
& \theta_{4}(u \mid \tau)=\theta_{4}(u)=\theta_{01}(u)=-i e^{\pi i \tau / 4+\pi i u} \theta_{1}(u+\tau / 2) .
\end{aligned}
$$

It is convenient to set $\theta_{a}\left(u_{1}, \ldots, u_{k}\right):=\theta_{a}\left(u_{1}\right) \cdots \theta_{a}\left(u_{k}\right)$ and $\theta_{a}(x \pm y):=\theta_{a}(x+y, x-y)$.

The $\theta_{1}(u)$-function is odd, $\theta_{1}(-u)=-\theta_{1}(u)$, and obeys the quasiperiodicity properties:

$$
\theta_{1}(u+1)=-\theta_{1}(u), \quad \theta_{1}(u+\tau)=-e^{-\pi i \tau-2 \pi i u} \theta_{1}(u)
$$

The short theta function

$$
\theta(z ; p):=(z ; p)_{\infty}\left(p z^{-1} ; p\right)_{\infty}, \quad z \in \mathbb{C}^{*},
$$

is related to $\theta_{1}(u)$ by the Jacobi triple product identity

$$
\theta_{1}(u)=i p^{1 / 8} e^{-\pi i u}(p ; p)_{\infty} \theta\left(e^{2 \pi i u} ; p\right) .
$$

We shall need the duplication formula for the $\theta_{1}$-function,

$$
\theta_{1}(2 u)=\frac{i p^{1 / 8}}{(p ; p)_{\infty}^{3}} \theta_{1}\left(u, u+\frac{1}{2}, u+\frac{\tau}{2}, u-\frac{1+\tau}{2}\right)
$$


and the addition formula

$$
\theta_{1}(u \pm x, v \pm y)-\theta_{1}(u \pm y, v \pm x)=\theta_{1}(x \pm y, u \pm v)
$$

or

$$
\theta\left(u x^{ \pm}, v y^{ \pm} ; p\right)-\theta\left(u y^{ \pm}, v x^{ \pm} ; p\right)=\frac{v}{x} \theta\left(x y^{ \pm}, u v^{ \pm} ; p\right),
$$

where $\theta\left(u x^{ \pm} ; p\right):=\theta\left(u x, u x^{-1} ; p\right):=\theta(u x ; p) \theta\left(u x^{-1} ; p\right)$.

The standard elliptic gamma function has the form

$$
\Gamma_{p, q}(z)=\prod_{j, k=0}^{\infty} \frac{1-z^{-1} p^{j+1} q^{k+1}}{1-z p^{j} q^{k}}, \quad|p|,|q|<1 .
$$

We set

$$
\begin{aligned}
\Gamma_{p, q}\left(t_{1}, \ldots, t_{k}\right) & :=\Gamma_{p, q}\left(t_{1}\right) \cdots \Gamma_{p, q}\left(t_{k}\right), \\
\Gamma_{p, q}\left(t z^{ \pm}\right) & :=\Gamma_{p, q}(t z) \Gamma_{p, q}\left(t z^{-1}\right), \quad \Gamma_{p, q}\left(z^{ \pm 2}\right):=\Gamma_{p, q}\left(z^{2}\right) \Gamma_{p, q}\left(z^{-2}\right) .
\end{aligned}
$$

This function satisfies two finite difference equations of the first order,

$$
\Gamma_{p, q}(q z)=\theta(z ; p) \Gamma_{p, q}(z), \quad \Gamma_{p, q}(p z)=\theta(z ; q) \Gamma_{p, q}(z),
$$

the reflection equation

$$
\Gamma_{p, q}(z) \Gamma_{p, q}(p q / z)=1
$$

the duplication formula

$$
\Gamma_{p, q}\left(z^{2}\right)=\Gamma_{p, q}\left(z,-z, q^{1 / 2} z,-q^{1 / 2} z, p^{1 / 2} z,-p^{1 / 2} z,(p q)^{1 / 2} z,-(p q)^{1 / 2} z\right),
$$

and the limit relation $\lim _{p \rightarrow 0} \Gamma_{p, q}(z)=1 /(z ; q)_{\infty}$.

With the help of three pairwise incommensurate parameters $\omega_{1,2,3} \in \mathbb{C}$, we define three base variables

$$
q=e^{2 \pi i \frac{\omega_{1}}{\omega_{2}}}, \quad p=e^{2 \pi i \frac{\omega_{3}}{\omega_{2}}}, \quad r=e^{2 \pi i \frac{\omega_{3}}{\omega_{1}}},
$$

and their particular modular transforms

$$
\tilde{q}=e^{-2 \pi i \frac{\omega_{2}}{\omega_{1}}}, \quad \tilde{p}=e^{-2 \pi i \frac{\omega_{2}}{\omega_{3}}}, \quad \tilde{r}=e^{-2 \pi i \frac{\omega_{1}}{\omega_{3}}} .
$$

The modified elliptic gamma function, which remains well defined for $\omega_{1} / \omega_{2}>0$ (i.e., $|q|=1$ ), has the form

$$
G(u ; \omega)=\Gamma_{p, q}\left(e^{2 \pi i \frac{u}{\omega_{2}}}\right) \Gamma_{\tilde{q}, r}\left(r e^{-2 \pi i \frac{u}{\omega_{1}}}\right)=e^{-\pi i P(u)} \Gamma\left(e^{-2 \pi i \frac{u}{\omega_{3}}} ; \tilde{r}, \tilde{p}\right),
$$

where $|p|,|r|<1$ and

$$
P\left(u+\sum_{m=1}^{3} \frac{\omega_{m}}{2}\right)=\frac{u\left(u^{2}-\frac{1}{4} \sum_{m=1}^{3} \omega_{m}^{2}\right)}{3 \omega_{1} \omega_{2} \omega_{3}} .
$$

It is fixed uniquely by the following three equations:

$$
\begin{aligned}
f\left(u+\omega_{1}\right) & =\theta\left(e^{2 \pi i u / \omega_{2}} ; p\right) f(u), \quad f\left(u+\omega_{2}\right)=\theta\left(e^{2 \pi i u / \omega_{1}} ; r\right) f(u), \\
f\left(u+\omega_{3}\right) & =e^{-\pi i B_{2,2}\left(u ; \omega_{1}, \omega_{2}\right)} f(u), \\
B_{2,2}\left(u ; \omega_{1}, \omega_{2}\right) & =\frac{u^{2}}{\omega_{1} \omega_{2}}-\frac{u}{\omega_{1}}-\frac{u}{\omega_{2}}+\frac{\omega_{1}}{6 \omega_{2}}+\frac{\omega_{2}}{6 \omega_{1}}+\frac{1}{2},
\end{aligned}
$$

together with the normalization $f\left(\sum_{m=1}^{3} \omega_{m} / 2\right)=1$. The reflection equation has the form $G(a ; \omega) G(b ; \omega)=1, a+b=\sum_{k=1}^{3} \omega_{k}$. A more detailed description of the elliptic gamma functions can be found in the reviews [13, or 19] and in the references therein. 


\section{$\S 2$. Continuous Biorthogonal functions}

We investigate elliptic hypergeometric integrals of the form

$$
\begin{aligned}
I^{(m)}\left(t_{1}, \ldots, t_{2 m+6}\right) & =\kappa \int_{\mathbb{T}} \frac{\prod_{j=1}^{2 m+6} \Gamma_{p, q}\left(t_{j} z^{ \pm}\right)}{\Gamma_{p, q}\left(z^{ \pm 2}\right)} \frac{d z}{z} \\
\prod_{j=1}^{2 m+6} t_{j} & =(p q)^{m+1}, \quad \kappa=\frac{(p ; p)_{\infty}(q ; q)_{\infty}}{4 \pi i}
\end{aligned}
$$

where $\mathbb{T}$ is the unit circle and $\left|t_{j}\right|<1$. For $m=0$, we obtain the elliptic beta integral 16]

$$
I^{(0)}\left(t_{1}, \ldots, t_{6}\right)=\prod_{1 \leq j<k \leq 6} \Gamma_{p, q}\left(t_{j} t_{k}\right)
$$

Using this formula, it is easy to verify the following recursion relation by a mere change of the order of integrations:

$$
\begin{aligned}
& I^{(m+1)}\left(t_{1}, \ldots, t_{2 m+8}\right)=\frac{\prod_{2 m+5 \leq k<l \leq 2 m+8} \Gamma_{p, q}\left(t_{k} t_{l}\right)}{\Gamma_{p, q}\left(\varepsilon_{m}^{2}\right)} \\
& \quad \times \kappa \int_{\mathbb{T}} \frac{\prod_{k=2 m+5}^{2 m+8} \Gamma_{p, q}\left(\varepsilon_{m}^{-1} t_{k} w^{ \pm}\right)}{\Gamma_{p, q}\left(w^{ \pm 2}\right)} I^{(m)}\left(t_{1}, \ldots, t_{2 m+4}, \varepsilon_{m} w, \varepsilon_{m} w^{-1}\right) \frac{d w}{w}
\end{aligned}
$$

where $\varepsilon_{m}=\sqrt{\prod_{k=2 m+5}^{2 m+8} t_{k} / p q}$. This leads to the $m$-tuple integral representation for $I^{(m)}$ similar to the Euler type integral representation for the general plain hypergeometric function ${ }_{m+1} F_{m}$; i.e., $I^{(m)}$ can be interpreted as an elliptic analog of the ${ }_{m+1} F_{m}$-function. The recurrence (2.2) is a special realization of an integral analog of the Bailey chains discovered in [18] (the Bailey chains technique is well known as a simplest tool for proving the Rogers-Ramanujan type identities [1]).

For $m=0$, the integrand on the right-hand side in (2.2) contains the elliptic beta integral, the computation of which yields

$$
V\left(t_{1}, \ldots, t_{8}\right)=\prod_{1 \leq k<l \leq 4} \Gamma_{p, q}\left(t_{k} t_{l}, t_{k+4} t_{l+4}\right) V\left(\varepsilon t_{1}, \ldots, \varepsilon t_{4}, \varepsilon^{-1} t_{5}, \ldots, \varepsilon^{-1} t_{8}\right)
$$

see [17, where $V(\underline{t})=I^{(1)}(\underline{t})$ is an elliptic analog of the Gauss hypergeometric function and $\varepsilon=\sqrt{t_{5} t_{6} t_{7} t_{8} / p q}=\sqrt{p q / t_{1} t_{2} t_{3} t_{4}}$. This is the key reflection, extending the obvious $S_{8}$-group of symmetries in the parameters of $V(\underline{t})$ to the Weyl group for the exceptional root system $E_{7}$.

For $m=1$, we obtain the identity

$$
\begin{aligned}
I^{(2)}\left(t_{1}, \ldots, t_{10}\right)= & \frac{\prod_{7 \leq k<l \leq 10} \Gamma_{p, q}\left(t_{k} t_{l}\right)}{\Gamma_{p, q}\left(\varepsilon_{1}^{2}\right)} \\
& \times \kappa \int_{\mathbb{T}} \frac{\prod_{k=7}^{10} \Gamma_{p, q}\left(\varepsilon_{1}^{-1} t_{k} w^{ \pm}\right)}{\Gamma_{p, q}\left(w^{ \pm 2}\right)} V\left(t_{1}, \ldots, t_{6}, \varepsilon_{1} w, \varepsilon_{1} w^{-1}\right) \frac{d w}{w},
\end{aligned}
$$

where $\varepsilon_{1}=\sqrt{t_{7} t_{8} t_{9} t_{10} / p q}$. After fixing parameters $t_{5} t_{7}=t_{6} t_{8}=p q$, the integral on the left-hand side reduces to the elliptic beta integral, and so, it can be computed explicitly. 
This yields the relation

$$
\begin{aligned}
\prod_{1 \leq k<l \leq 4} \Gamma_{p, q}\left(t_{k} t_{l}\right) & \prod_{k=1}^{4} \Gamma_{p, q}\left(t_{k} t_{9}, t_{k} t_{10}\right)=\frac{\prod_{7 \leq k<l \leq 10} \Gamma_{p, q}\left(t_{k} t_{l}\right)}{\Gamma_{p, q}\left(t_{9} t_{10}\right) \Gamma_{p, q}\left(\varepsilon_{1}^{2}\right)} \\
\times & \kappa \int_{\mathbb{T}} \frac{\prod_{j=7}^{10} \Gamma_{p, q}\left(\varepsilon_{1}^{-1} t_{j} w^{ \pm}\right)}{\Gamma_{p, q}\left(w^{ \pm 2}\right)} V\left(t_{1}, \ldots, t_{4}, p q / t_{7}, p q / t_{8}, \varepsilon_{1} w, \varepsilon_{1} w^{-1}\right) \frac{d w}{w},
\end{aligned}
$$

where $t_{1} t_{2} t_{3} t_{4} t_{9} t_{10}=p q$. Now we denote $t_{9}=s \xi, t_{10}=s \xi^{-1}$ and obtain

$$
\begin{aligned}
\prod_{1 \leq k<l \leq 4} \Gamma_{p, q}\left(t_{k} t_{l}\right) & \prod_{j=1}^{4} \Gamma_{p, q}\left(t_{j} s \xi^{ \pm}\right)=\frac{\Gamma_{p, q}\left(t_{7} s \xi^{ \pm}, t_{8} s \xi^{ \pm}, t_{7} t_{8}\right)}{\Gamma_{p, q}\left(\varepsilon_{1}^{2}\right)} \\
& \times \kappa \int_{\mathbb{T}} \frac{\Gamma_{p, q}\left(\varepsilon_{1}^{-1} t_{7} w^{ \pm}, \varepsilon_{1}^{-1} t_{8} w^{ \pm}, \varepsilon_{1}^{-1} s \xi^{ \pm} w^{ \pm}\right)}{\Gamma_{p, q}\left(w^{ \pm 2}\right)} \\
& \times V\left(t_{1}, \ldots, t_{4}, p q / t_{7}, p q / t_{8}, \varepsilon_{1} w, \varepsilon_{1} w^{-1}\right) \frac{d w}{w}
\end{aligned}
$$

where $t_{1} t_{2} t_{3} t_{4} s^{2}=p q$ and $\varepsilon_{1}=s \sqrt{t_{7} t_{8} / p q}$. After the replacements

$$
t_{1}=c, \quad t_{2}=d, \quad t_{3}=s^{-1} \sqrt{\frac{p q}{c d}} x, \quad t_{4}=s^{-1} \sqrt{\frac{p q}{c d}} x^{-1}, \quad t_{7}=a, \quad t_{8}=b,
$$

the condition $t_{1} t_{2} t_{3} t_{4} s^{2}=p q$ is satisfied automatically and $\varepsilon_{1}=s \sqrt{a b / p q}$. Introducing the basis vectors

$$
\phi(w ; a, b \mid \xi ; s)=\Gamma_{p, q}\left(s a \xi^{ \pm}, s b \xi^{ \pm}, \sqrt{\frac{p q}{a b}} w^{ \pm} \xi^{ \pm}\right),
$$

we can rewrite the key relation (2.5) as

$$
\phi(x ; c, d \mid \xi ; s)=\kappa \int_{\mathbb{T}} R(c, d, a, b ; x, w \mid s) \phi(w ; a, b \mid \xi ; s) \frac{d w}{w},
$$

where, after the substitution $s=\sqrt{p q / \rho}$,

$$
\begin{aligned}
R(c, d, a, b ; x, w \mid s)= & \frac{\Gamma_{p, q}\left(a b, \sqrt{\frac{a \rho}{b}} w^{ \pm}, \sqrt{\frac{b \rho}{a}} w^{ \pm}\right)}{\Gamma_{p, q}\left(c d, \sqrt{\frac{c \rho}{d}} x^{ \pm}, \sqrt{\frac{d \rho}{c}} x^{ \pm}\right)} \frac{1}{\Gamma_{p, q}\left(\frac{a b}{\rho}, \frac{\rho}{c d}, w^{ \pm 2}\right)} \\
& \times V\left(c, d, \sqrt{\frac{\rho}{c d}} x, \sqrt{\frac{\rho}{c d}} x^{-1}, \frac{p q}{a}, \frac{p q}{b}, \sqrt{\frac{a b}{\rho}} w, \sqrt{\frac{a b}{\rho}} w^{-1}\right) .
\end{aligned}
$$

Applying the transformation (2.3), we can transform this function to

$$
\begin{aligned}
R(c, d, a, b ; x, w \mid s)= & \frac{1}{\Gamma_{p, q}\left(\frac{p q}{a b}, \frac{a b}{p q}, w^{ \pm 2}\right)} \\
& \times V\left(s c, s d, \sqrt{\frac{p q}{c d}} x, \varepsilon \sqrt{\frac{p q}{c d}} x^{-1}, \frac{p q}{a s}, \frac{p q}{b s}, \sqrt{\frac{a b}{p q}} w, \sqrt{\frac{a b}{p q}} w^{-1}\right) .
\end{aligned}
$$

The kernel $R$ does not depend on the variable $\xi$. Relation (2.7) looks therefore like a rotation in some function space spanned by the basis vectors $\phi$, which is performed by the "matrix" $R$ with the continuous indices $x$ and $w$.

We can denote $\xi=e^{i \chi}, w=e^{i \theta}$, and $x=e^{i \varphi}$ and let $\chi, \theta$, and $\varphi$ take real values only. Then, because of the symmetries

$$
\phi(w ; a, b \mid \xi ; s)=\phi\left(w^{-1} ; a, b \mid \xi ; s\right)=\phi\left(w ; a, b \mid \xi^{-1} ; s\right),
$$


we see that this function depends actually on the variables $y:=\cos \theta$ and $\cos \chi$. Similarly, the function

$$
R(a, b, c, d ; x, w \mid s)=R\left(a, b, c, d ; x^{-1}, w \mid s\right)=R\left(a, b, c, d ; x, w^{-1} \mid s\right)
$$

depends on $y$ and $\cos \varphi$. Using the elementary relation

$$
\int_{\mathbb{T}} f(\cos \theta) \frac{d w}{i w}=\int_{0}^{2 \pi} f(\cos \theta) d \theta=2 \int_{-1}^{1} f(y) \frac{d y}{\sqrt{1-y^{2}}}
$$

we can write

$$
\phi(x ; c, d \mid \xi ; s)=2 i \kappa \int_{-1}^{1} R\left(c, d, a, b ; x, e^{i \theta} \mid s\right) \phi\left(e^{i \theta} ; a, b \mid \xi ; s\right) \frac{d y}{\sqrt{1-y^{2}}} .
$$

Letting $c \rightarrow a$ and $d \rightarrow b$ and denoting $v=\cos \varphi$, we obtain the relation

$$
\lim _{c \rightarrow a, d \rightarrow b} R\left(c, d, a, b ; e^{i \varphi}, e^{i \theta}\right)=\frac{2 \pi \sqrt{1-y^{2}}}{(p ; p)_{\infty}(q ; q)_{\infty}} \delta(v-y)
$$

in the distributional sense. Formally, this relation can be rewritten as

$$
\begin{array}{r}
V\left(a, b, \sqrt{\frac{\rho}{a b}} e^{i \varphi}, \sqrt{\frac{\rho}{a b}} e^{-i \varphi}, \frac{p q}{a}, \frac{p q}{b}, \sqrt{\frac{a b}{\rho}} e^{i \theta}, \sqrt{\frac{a b}{\rho}} e^{-i \theta}\right) \\
=\Gamma_{p, q}\left(\frac{a b}{\rho}, \frac{\rho}{a b}, e^{ \pm 2 i \theta}\right) \frac{2 \pi \sqrt{1-y^{2}}}{(p ; p)_{\infty}(q ; q)_{\infty}} \delta(v-y) .
\end{array}
$$

Using identity (2.7) twice with different choices of parameters on the right-hand side, we obtain immediately the self-reproducing property for the kernel

$$
\kappa \int_{\mathbb{T}} R(a, b, c, d ; x, w \mid s) R(c, d, e, f ; w, z \mid s) \frac{d w}{w}=R(a, b, e, f ; x, z \mid s)
$$

and the biorthogonality relation

$$
\int_{-1}^{1} R\left(a, b, c, d ; e^{i \varphi}, e^{i \theta} \mid s\right) R\left(c, d, a, b ; e^{i \theta}, e^{i \varphi^{\prime}} \mid s\right) \frac{d y}{\sqrt{1-y^{2}}}=\frac{\sqrt{1-v^{2}}}{(2 i \kappa)^{2}} \delta\left(v-v^{\prime}\right)
$$

where $v=\cos \varphi$ and $v^{\prime}=\cos \varphi^{\prime}$ can be viewed as continuous spectral variables. Substituting the explicit expressions for the $R$-functions, we rewrite the biorthogonality relation as

$$
\begin{aligned}
\int_{-1}^{1} & \frac{1}{\Gamma_{p, q}\left(e^{ \pm 2 i \theta}\right)} V\left(a, b, \sqrt{\frac{\rho}{a b}} e^{i \varphi}, \sqrt{\frac{\rho}{a b}} e^{-i \varphi}, \frac{p q}{c}, \frac{p q}{d}, \sqrt{\frac{c d}{\rho}} e^{i \theta}, \sqrt{\frac{c d}{\rho}} e^{-i \theta}\right) \\
& \times V\left(c, d, \sqrt{\frac{\rho}{c d}} e^{i \theta}, \sqrt{\frac{\rho}{c d}} e^{-i \theta}, \frac{p q}{a}, \frac{p q}{b}, \sqrt{\frac{a b}{\rho}} e^{i \varphi^{\prime}}, \sqrt{\frac{a b}{\rho}} e^{-i \varphi^{\prime}}\right) \frac{d y}{\sqrt{1-y^{2}}} \\
= & \Gamma_{p, q}\left(\frac{a b}{\rho}, \frac{\rho}{a b}, \frac{c d}{\rho}, \frac{\rho}{c d}, e^{ \pm 2 i \varphi}\right) \frac{\sqrt{1-v^{2}}}{(2 i \kappa)^{2}} \delta\left(v-v^{\prime}\right) .
\end{aligned}
$$

\section{§3. A scalar PRODUCT AND Biorthogonality of THE BASIS VECTORS}

Now it is necessary to determine the Hilbert space content of the construction described above. Desirably, the $V$-function should be given by a scalar product of two basis vectors. 
Multiplying (2.7) by $\phi\left(z ; p q / e, p q / f \mid \xi ; s^{-1}\right)$ and integrating over $\xi$ against the measure $\kappa \int_{\mathbb{T}} \Gamma_{p, q}^{-1}\left(\xi^{ \pm 2}\right) d \xi / \xi$, on the left-hand side we obtain

$$
\kappa \int_{\mathbb{T}} \frac{\phi\left(z ; \frac{p q}{e}, \frac{p q}{f} \mid \xi ; s^{-1}\right) \phi(x ; c, d \mid \xi ; s)}{\Gamma_{p, q}\left(\xi^{ \pm 2}\right)} \frac{d \xi}{\xi}=V\left(s t_{1}, \ldots, s t_{4}, s^{-1} t_{5}, \ldots, s^{-1} t_{8}\right),
$$

where $t_{1}=c, t_{2}=d, t_{3,4}=x^{ \pm 1} s^{-1} \sqrt{p q / c d}$ and $t_{5}=p q / e, t_{6}=p q / f, t_{7,8}=$ $z^{ \pm 1} s \sqrt{e f / p q}$. On the right-hand side, we find

$$
\kappa \int_{\mathbb{T}} R(c, d, a, b ; x, w \mid s) V\left(s t_{1}^{\prime}, \ldots, s t_{4}^{\prime}, s^{-1} t_{5}, \ldots, s^{-1} t_{8}\right) \frac{d w}{w},
$$

where $t_{1}^{\prime}=a, t_{2}^{\prime}=b, t_{3,4}^{\prime}=w^{ \pm 1} s^{-1} \sqrt{p q / a b}$. If we apply the $E_{7}$-transformation (2.3) to $V$-functions on both sides of the identity obtained, we arrive at relation (2.11), which shows that our consideration is self-consistent.

Letting $e \rightarrow c, f \rightarrow d$, and applying (2.10) to the right-hand side of (3.1), we find the biorthogonality relation

$$
\begin{aligned}
\kappa \int_{\mathbb{T}} \frac{\phi\left(e^{i \varphi^{\prime}} ; \frac{p q}{c}, \frac{p q}{d} \mid \xi ; s^{-1}\right) \phi\left(e^{i \varphi} ; c, d \mid \xi ; s\right)}{\Gamma_{p, q}\left(\xi^{ \pm 2}\right)} \frac{d \xi}{\xi} \\
=\Gamma_{p, q}\left(\frac{p q}{c d}, \frac{c d}{p q}, e^{ \pm 2 i \varphi}\right) \sqrt{1-v^{2}} \delta\left(v-v^{\prime}\right) .
\end{aligned}
$$

The latter result inspires the introduction of the following inner product. For two functions of $z \in \mathbb{C}$ with the property $\psi(z)=\psi\left(z^{-1}\right)$, we define

$$
\langle\chi(z), \psi(z)\rangle=\kappa \int_{\mathbb{T}} \frac{\chi(z) \psi(z)}{\Gamma_{p, q}\left(z^{ \pm 2}\right)} \frac{d z}{z} .
$$

The reflection equation for the elliptic gamma function implies that, for $z \in \mathbb{T}$ and real $(p, q)$-variables,

$$
\frac{1}{\Gamma_{p, q}\left(z^{ \pm 2}\right)}=\theta\left(z^{2} ; p\right) \theta\left(z^{-2} ; q\right)=\left|\left(1-z^{2}\right)\left(z^{2} ; p\right)_{\infty}\left(q z^{2} ; q\right)_{\infty}\right|^{2}
$$

i.e., our measure is positive definite. Similarly, for $p=q^{*}$, we also obtain a positive measure,

$$
\frac{1}{\Gamma_{p, q}\left(z^{ \pm 2}\right)}=\left|\theta\left(z^{2} ; p\right)\right|^{2}=\left|\left(z^{2} ; p\right)_{\infty}\left(q z^{2} ; q\right)_{\infty}\right|^{2}
$$

We introduce the finite difference operator 9, 11]

$$
D_{R}(a, b, c, d ; p ; q):=\frac{\theta(a z, b z, c z, d z ; p)}{\theta\left(z^{2} ; p\right)} T_{z, q}^{1 / 2}+\frac{\theta\left(a z^{-1}, b z^{-1}, c z^{-1}, d z^{-1} ; p\right)}{\theta\left(z^{-2} ; p\right)} T_{z, q}^{-1 / 2}
$$

where $T_{z, q}^{ \pm 1 / 2} f(z)=f\left(q^{ \pm 1 / 2} z\right)$ is a $q$-shift operator. The operator conjugate to $D_{R}$ is defined by

$$
\left\langle\chi, D_{R}(a, b, c, d ; p ; q) \psi\right\rangle=\left\langle D_{R}^{*}(a, b, c, d ; p ; q) \chi, \psi\right\rangle .
$$

Assuming that functions $\psi(z)$ and $\chi(z)$ have no singularities in the annulus $|q|^{1 / 2}<|z|<$ $|q|^{-1 / 2}$, we find

$$
D_{R}^{*}(a, b, c, d ; p ; q)=D_{R}\left(\frac{p q^{1 / 2}}{a}, \frac{p q^{1 / 2}}{b}, \frac{p q^{1 / 2}}{c}, \frac{p q^{1 / 2}}{d} ; p ; q\right) .
$$

Clearly, $D_{R}^{* *}=D_{R}$. For further considerations, it is convenient to scale one of the parameters by $p$ and define $D(a, b, c, d ; p ; q):=-a D_{R}(p a, b, c, d ; p ; q)$, so that

$$
D(a, b, c, d ; p ; q)=\frac{\theta(a z, b z, c z, d z ; p)}{z \theta\left(z^{2} ; p\right)} T_{z, q}^{1 / 2}+\frac{\theta\left(a z^{-1}, b z^{-1}, c z^{-1}, d z^{-1} ; p\right)}{z^{-1} \theta\left(z^{-2} ; p\right)} T_{z, q}^{-1 / 2}
$$


and

$$
D^{*}(a, b, c, d ; p ; q)=\frac{c d}{q^{1 / 2}} D\left(\frac{p q^{1 / 2}}{a}, \frac{p q^{1 / 2}}{b}, \frac{q^{1 / 2}}{c}, \frac{q^{1 / 2}}{d} ; p ; q\right) .
$$

Now we look for solutions of the generalized eigenvalue problem for $D$-operators with the fixed product $\rho:=a b c d$,

$$
D(a, b, c, d ; p ; q) f(z)=\lambda D\left(a, b, c^{\prime}, d^{\prime} ; p ; q\right) f(z),
$$

where $c d=c^{\prime} d^{\prime}$. We parametrize the spectral variable $\lambda$ by an elliptic function of the second order,

$$
\lambda \equiv \lambda(w)=\frac{\theta(w \sqrt{c / d}, w \sqrt{d / c} ; p)}{\theta\left(w \sqrt{c^{\prime} / d^{\prime}}, w \sqrt{d^{\prime} / c^{\prime}} ; p\right)},
$$

where $w \in \mathbb{C}$ is an arbitrary parameter, and indicate the dependence on all parameters in $f(z)$,

$$
f(z):=f\left(z ; w ; q^{1 / 2} a, q^{1 / 2} b ; \rho\right),
$$

where the parameters $a$ and $b$ are scaled by $q^{1 / 2}$ for convenience. Then equation (3.5) can be rewritten as

$$
\begin{aligned}
& \frac{f\left(q^{1 / 2} z ; w ; q^{1 / 2} a, q^{1 / 2} b ; \rho\right)}{f\left(q^{-1 / 2} z ; w ; q^{1 / 2} a, q^{1 / 2} b ; \rho\right)}=-z^{2} \frac{\theta\left(z^{2}, a z^{-1}, b z^{-1} ; p\right)}{\theta\left(z^{-2}, a z, b z ; p\right)} \\
& \quad \times \frac{\theta\left(c z^{-1}, d z^{-1}, w \sqrt{c^{\prime} / d^{\prime}}, w \sqrt{d^{\prime} / c^{\prime}} ; p\right)-\theta\left(c^{\prime} z^{-1}, d^{\prime} z^{-1}, w \sqrt{c / d}, w \sqrt{d / c} ; p\right)}{\theta\left(c z, d z, w \sqrt{c^{\prime} / d^{\prime}}, w \sqrt{d^{\prime} / c^{\prime}} ; p\right)-\theta\left(c^{\prime} z, d^{\prime} z, w \sqrt{c / d}, w \sqrt{d / c} ; p\right)},
\end{aligned}
$$

which, after application of the addition formula for $\theta(z ; p)$-functions, reduces to

$$
\frac{f\left(q^{1 / 2} z ; w ; q^{1 / 2} a, q^{1 / 2} b ; \rho\right)}{f\left(q^{-1 / 2} z ; w ; q^{1 / 2} a, q^{1 / 2} b ; \rho\right)}=z^{4} \frac{\theta\left(a z^{-1}, b z^{-1}, \sqrt{c d} w^{ \pm} z^{-1} ; p\right)}{\theta\left(a z, b z, \sqrt{c d} w^{ \pm} z ; p\right)} .
$$

A particular solution of this equation, valid for $|p|,|q|<1$, has the form

$$
f(z ; w ; a, b ; \rho)=\Gamma_{p, q}\left(\frac{p q}{a} z^{ \pm}, \frac{p q}{b} z^{ \pm}, \sqrt{\frac{a b}{\rho}} w^{ \pm} z^{ \pm}\right) .
$$

The general solution for $|q|<1$ is obtained from (3.7) after multiplication by an arbitrary function $\varphi(z)$ satisfying $\varphi(q z)=\varphi(z)$. The case where $|q|=1$ will be considered below, but the regime $|q|>1$ will be omitted for brevity, because it turns out to be equivalent to the case of $|q|<1$ after a change of parameters.

Our particular solution depends only on three parameters $a, b$, and $\rho$; i.e., two variables, say, $c$ and $c^{\prime}$, drop out, being plain gauge parameters. A special choice of parameters reduces $f(z ; w ; a, b ; \rho)$ to products of theta functions,

$$
f\left(z ; q^{k} \sqrt{\frac{a}{b q^{N}}} ; a, b ; q^{-N}\right)=\prod_{j=0}^{k-1} \theta\left(q^{j} a z^{ \pm} ; p\right) \prod_{j=0}^{N-k-1} \theta\left(q^{j} b z^{ \pm} ; p\right), \quad k, N \in \mathbb{N},
$$

which coincide with the intertwining vectors of Takebe [21] and the basis vectors used by Rosengren in [11, 12.

Since the function $f(z ; w ; a, b ; \rho)$ is symmetric in $p$ and $q$, we can demand it satisfy yet another generalized eigenvalue problem, obtained from (3.10) by permutation of $p$ and $q$ :

$$
\begin{aligned}
& D(a, b, c, d ; q ; p) f\left(z ; w ; p^{1 / 2} a, p^{1 / 2} b ; \rho\right) \\
& \quad=\frac{\theta(w \sqrt{c / d}, w \sqrt{d / c} ; q)}{\theta\left(w \sqrt{c^{\prime} / d^{\prime}}, w \sqrt{d^{\prime} / c^{\prime}} ; q\right)} D\left(a, b, c^{\prime}, d^{\prime} ; q ; p\right) f\left(z ; w ; p^{1 / 2} a, p^{1 / 2} b ; \rho\right),
\end{aligned}
$$


where $c d=c^{\prime} d^{\prime}$. For incommensurate $p$ and $q$ (which will be assumed throughout the paper), this requirement strongly restricts the space of solutions of our eigenvalue problem, because the equations $\varphi(q z)=\varphi(p z)=\varphi(z)$ are satisfied only by a constant function. Our basis vectors $f(z ; w ; a, b ; \rho)$ thus become fixed uniquely up to a constant factor, which we set equal to 1 for definiteness.

The function (3.7) satisfies another type of the generalized eigenvalue problem,

$$
D(a, b, c, d ; p, q) f\left(z ; w ; q^{1 / 2} a, q^{1 / 2} b ; \rho\right)=d^{-1} \theta\left(w^{ \pm} \sqrt{d / c}, c d ; p\right) f(z ; w ; a, b ; \rho) .
$$

Indeed, dividing this relation by $f(z ; w ; a, b)$, on the left-hand side we obtain

$$
\begin{aligned}
& \frac{\theta(a z, b z, c z, d z ; p)}{z \theta\left(z^{2} ; p\right)} \frac{f\left(q^{1 / 2} z ; w ; q^{1 / 2} a, q^{1 / 2} b ; \rho\right)}{f(z ; w ; a, b ; \rho)} \\
&+\frac{\theta\left(a z^{-1}, b z^{-1}, c z^{-1}, d z^{-1} ; p\right)}{z^{-1} \theta\left(z^{-2} ; p\right)} \frac{f\left(q^{-1 / 2} z ; w ; q^{1 / 2} a, q^{1 / 2} b ; \rho\right)}{f(z ; w ; a, b ; \rho)} .
\end{aligned}
$$

After substitution of the explicit form of $f(z ; w ; a, b ; \rho)$, this expression reduces to

$$
\frac{\theta\left(c z, d z, w^{ \pm} z \sqrt{a b / \rho} ; p\right)-\theta\left(c^{-1} z, d^{-1} z, w^{ \pm} z \sqrt{\rho / a b} ; p\right)}{z \theta\left(z^{2} ; p\right)} .
$$

The addition formula (1.2) with $u=z \sqrt{c d}, x=\sqrt{c / d}, v=z \sqrt{a b / \rho}, y=w$ yields an expression required for the right-hand side in (3.10). Identity (3.5) is easily deduced from (3.10) by observing that $f(z ; w ; a, b ; \rho)$ does not change after the replacement of $c$ and $d$ by $c^{\prime}$ and $d^{\prime}$ with the constraint $c d=c^{\prime} d^{\prime}$.

The commutation relation [10]

$$
\begin{aligned}
& D\left(a, b, c^{\prime}, d^{\prime} ; p ; q\right) D\left(q^{1 / 2} a, q^{1 / 2} b, q^{-1 / 2} c, q^{-1 / 2} d ; p ; q\right) \\
& \quad=D(a, b, c, d ; p ; q) D\left(q^{1 / 2} a, q^{1 / 2} b, q^{-1 / 2} c^{\prime}, q^{-1 / 2} d^{\prime} ; p ; q\right),
\end{aligned}
$$

where $c d=c^{\prime} d^{\prime}$, is a simple consequence of (3.10). Indeed, the action of both sides of this formula on the function $f(z ; w ; q a, q b ; \rho)$ yields the same result. The straightforward verification of the identity is also possible (the coincidence of the terms in front of the operators $T_{z, q}^{ \pm 1}$ is evident, and the scalar terms coincide after an application of the addition formula for theta functions).

Consider the generalized eigenvalue problem for the conjugate operators

$$
D^{*}(a, b, c, d ; p ; q) g(z)=\lambda(v) D^{*}\left(a, b, c^{\prime}, d^{\prime} ; p ; q\right) g(z),
$$

where $c d=c^{\prime} d^{\prime}$ and $\lambda(v)$ is a new spectral variable. Evidently, this equation is obtained from (3.5) after the replacements $a \rightarrow \tilde{a}=p q^{1 / 2} / a, b \rightarrow \tilde{b}=p q^{1 / 2} / b, c \rightarrow \tilde{c}=q^{1 / 2} / c$, $d \rightarrow \tilde{d}=q^{1 / 2} / d$, and $w \rightarrow v$. Thus, we find its particular solution

$$
g(z):=g(z ; v ; a, b ; \rho)=\Gamma_{p, q}\left(a z^{ \pm}, b z^{ \pm}, \sqrt{\frac{\rho}{a b}} v^{ \pm} z^{ \pm}\right) .
$$

The biorthogonality relation in question emerges as follows:

$$
\begin{aligned}
0= & \left\langle\left(D^{*}(a, b, c, d ; p ; q)-\lambda(v) D^{*}\left(a, b, c^{\prime}, d^{\prime} ; p ; q\right)\right) g(z ; v ; a, b ; \rho), f\left(z ; w ; q^{1 / 2} a, q^{1 / 2} b ; \rho\right)\right\rangle \\
= & \langle g(z ; v ; a, b ; \rho), \\
& \left.\quad\left(D(a, b, c, d ; p ; q)-\lambda(v) D\left(a, b, c^{\prime}, d^{\prime} ; p ; q\right)\right) f\left(z ; w ; q^{1 / 2} a, q^{1 / 2} ; \rho\right)\right\rangle \\
= & \left(1-\frac{\lambda(v)}{\lambda(w)}\right)\left\langle g(z ; v ; a, b ; \rho), D(a, b, c, d ; p ; q) f\left(z ; w ; q^{1 / 2} a, q^{1 / 2} b ; \rho\right)\right\rangle \\
= & \left(1-\frac{\lambda(v)}{\lambda(w)}\right) d^{-1} \theta\left(w^{ \pm} \sqrt{d / c}, c d ; p\right)\langle g(z ; v ; a, b ; \rho), f(z ; w ; a, b ; \rho)\rangle .
\end{aligned}
$$


For $\lambda(v) \neq \lambda(w)$, we find $\langle g(z ; v ; a, b ; \rho), f(z ; w ; a, b ; \rho)\rangle=0$, which essentially coincides with (2.10). Restrictions on the parameters needed for conjugating of the operator $D$ without crossing the singularities are $|p|<|a|,|b|<|q|^{1 / 2}$ and $|\rho / a b q|<|v|^{2}<|a b q / \rho|$, $|a b / \rho q|<|w|^{2}<|\rho q / a b|$.

To conclude, solutions of the generalized eigenvalue problems for the $D$-operator and its conjugate determine a basis rotated by the $R$-function and its dual. An overlap of these bases determines the $V$-function:

$$
\kappa \int_{\mathbb{T}} \frac{g(z ; v ; a, b ; e) f(z ; w ; c, d ; e)}{\Gamma_{p, q}\left(z^{ \pm 2}\right)} \frac{d z}{z}=V\left(a, b, \sqrt{\frac{e}{a b}} v^{ \pm}, \frac{p q}{c}, \frac{p q}{d}, \sqrt{\frac{c d}{e}} w^{ \pm},\right),
$$

where $V\left(\ldots, a x^{ \pm}, \ldots\right)=V\left(\ldots, a x, a x^{-1}, \ldots\right)$.

Also, we can rewrite the key relation (2.7) in a compact form:

$$
\begin{aligned}
& \Gamma_{p, q}\left(\alpha z^{ \pm} \xi^{ \pm}, \beta x^{ \pm} \xi^{ \pm}\right)=\kappa \int_{\mathbb{T}} r(\alpha, \beta, \gamma, \delta ; z, x ; t, w) \Gamma_{p, q}\left(\gamma t^{ \pm} \xi^{ \pm}, \delta w^{ \pm} \xi^{ \pm}\right) \frac{d w}{w} \\
& r(\alpha, \beta, \gamma, \delta ; z, x ; t, w)=\frac{1}{\Gamma_{p, q}\left(\delta^{ \pm 2}, w^{ \pm 2}\right)} V\left(\beta x^{ \pm}, \alpha z^{ \pm}, \frac{p q}{\gamma} t^{ \pm}, \frac{w^{ \pm}}{\delta}\right)
\end{aligned}
$$

where $\alpha \beta=\gamma \delta$.

\section{§4. ElLiptic HYPERGEOMETRIC EQUATION}

We recall the derivation of the elliptic hypergeometric equation together with its general solution (see [19, 20]). Repeating the transformation (2.3) with the parameters $\varepsilon t_{3}$, $\varepsilon t_{4}, \varepsilon^{-1} t_{5}, \varepsilon^{-1} t_{6}$ that play the role of $t_{1}, \ldots, t_{4}$, and permuting $t_{3}, t_{4}$ with $t_{5}, t_{6}$ in the result, we find

$$
V(\underline{t})=\prod_{j, k=1}^{4} \Gamma_{p, q}\left(t_{j} t_{k+4}\right) V\left(\sqrt{T} / t_{1}, \ldots, \sqrt{T} / t_{4}, \sqrt{U} / t_{5}, \ldots, \sqrt{U} / t_{8}\right),
$$

where $T=t_{1} t_{2} t_{3} t_{4}$ and $U=t_{5} t_{6} t_{7} t_{8}$. Equating the right-hand sides of (2.3) and (4.1), we obtain the third symmetry transformation

$$
V(\underline{s})=\prod_{1 \leq j<k \leq 8} \Gamma_{p, q}\left(s_{j} s_{k}\right) V\left(\sqrt{p q} / s_{1}, \ldots, \sqrt{p q} / s_{8}\right) .
$$

All these relations represent different Weyl group transformations for the exceptional root system $E_{7}$ in the parameter space.

If we set $v=t_{6}, x=t_{7}, y=z$, and $u=t_{8}$ in the addition formula (1.2), then it becomes equivalent to the equation

$$
\begin{array}{r}
t_{7} \theta\left(t_{8} t_{7}^{ \pm} ; p\right) \Delta\left(z, t_{1}, \ldots, t_{5}, q t_{6}, t_{7}, t_{8}\right)-t_{7} \theta\left(t_{6} t_{7}^{ \pm} ; p\right) \Delta\left(z, t_{1}, \ldots, t_{7}, q t_{8}\right) \\
=t_{6} \theta\left(t_{8} t_{6}^{ \pm} ; p\right) \Delta\left(z, t_{1}, \ldots, t_{6}, q t_{7}, t_{8}\right),
\end{array}
$$

where $\Delta(z, \underline{t})=\prod_{k=1}^{8} \Gamma_{p, q}\left(t_{k} z^{ \pm}\right) / \Gamma_{p, q}\left(z^{ \pm 2}\right)$ is the $V$-function's kernel. Integration over $z$ along an appropriate contour $C$ yields

$$
t_{7} \theta\left(t_{8} t_{7}^{ \pm} ; p\right) V\left(q t_{6}\right)-t_{6} \theta\left(t_{8} t_{6}^{ \pm} ; p\right) V\left(q t_{7}\right)=t_{7} \theta\left(t_{6} t_{7}^{ \pm} ; p\right) V\left(q t_{8}\right),
$$

where the $V\left(q t_{j}\right)$ denote $V$-functions with the parameters $t_{j}$ replaced by $q t_{j}$ and the balancing condition $\prod_{j=1}^{8} t_{j}=p^{2} q$. Substituting the transformation (4.2) in this equation, 
we obtain

$$
\begin{gathered}
t_{6} \theta\left(\frac{t_{7}}{t_{8}} ; p\right) \prod_{k=1}^{5} \theta\left(t_{6} t_{k} ; p\right) V\left(q t_{7}, q t_{8}\right)-t_{7} \theta\left(\frac{t_{6}}{t_{8}} ; p\right) \prod_{k=1}^{5} \theta\left(t_{7} t_{k} ; p\right) V\left(q t_{6}, q t_{8}\right) \\
=t_{6} \theta\left(\frac{t_{7}}{t_{6}} ; p\right) \prod_{k=1}^{5} \theta\left(t_{8} t_{k} ; p\right) V\left(q t_{6}, q t_{7}\right)
\end{gathered}
$$

where $\prod_{j=1}^{8} t_{j}=p^{2}$.

Now we permute the parameters $t_{5}$ and $t_{8}$ in the last equation, change the parameters $t_{5}, t_{7} \rightarrow t_{5} / q, t_{7} / q$, and exclude the function $V\left(q t_{6}, q^{-1} t_{7}\right)$ with the help of identity (4.3), where the parameter $t_{7}$ is replaced with $t_{7} / q$. The resulting relation looks like this:

$$
\begin{aligned}
\frac{\theta\left(t_{7} t_{6}^{ \pm} / q ; p\right)}{\theta\left(t_{8} t_{6}^{ \pm} ; p\right)} & V\left(q^{-1} t_{7}, q t_{8}\right)-V(\underline{t}) \\
= & \frac{\theta\left(t_{7} / q t_{6}, t_{5} t_{8} / q, q t_{8} / t_{7} ; p\right)}{\theta\left(t_{8} t_{6}^{ \pm}, q t_{6} / t_{5} ; p\right)} \prod_{j=1}^{4} \frac{\theta\left(t_{5} t_{j} / q ; p\right)}{\theta\left(t_{7} t_{j} / q ; p\right)} V\left(q^{-1} t_{5}, q t_{6}\right) \\
& -\frac{\theta\left(q t_{8} / t_{7}, t_{7} / t_{5} ; p\right)}{\theta\left(t_{8} / t_{6}, q t_{6} / t_{5} ; p\right)} \prod_{j=1}^{4} \frac{\theta\left(t_{6} t_{j} ; p\right)}{\theta\left(t_{7} t_{j} / q ; p\right)} V(\underline{t}) .
\end{aligned}
$$

Multiplying it by the function

$$
\mathcal{A}\left(t_{1}, \ldots, t_{8} ; p\right)=\frac{\theta\left(t_{8} / q t_{6}, t_{6} t_{8}, t_{6} / t_{8} ; p\right)}{\theta\left(t_{8} / t_{7}, t_{7} / q t_{8}, t_{7} t_{8} / q ; p\right)} \prod_{j=1}^{5} \frac{\theta\left(t_{7} t_{j} / q ; p\right)}{\theta\left(t_{6} t_{j} ; p\right)}
$$

and symmetrizing in the parameters $t_{7}$ and $t_{8}$, we get the elliptic hypergeometric equation

$$
\mathcal{A}\left(t_{1}, \ldots, t_{7}, t_{8} ; p\right)\left(\frac{\theta\left(t_{7} t_{6}^{ \pm} / q ; p\right)}{\theta\left(t_{8} t_{6}^{ \pm} ; p\right)} V\left(q^{-1} t_{7}, q t_{8}\right)-V(\underline{t})\right)+\left(t_{7} \leftrightarrow t_{8}\right)+V(\underline{t})=0
$$

which was deduced in 19 in a slightly different way.

We set $t_{7}=c x^{-1}, t_{8}=c x$ and denote

$$
\mathcal{D}\left(t_{1}, \ldots, t_{8}\right)=a\left(t_{1}, \ldots, t_{7}, t_{8}\right)\left(T_{x, q}-1\right)+a\left(t_{1}, \ldots, t_{8}, t_{7}\right)\left(T_{x, q}^{-1}-1\right)+\kappa\left(t_{1}, \ldots, t_{8}\right),
$$

where $T_{x, q} f(x)=f(q x)$ and

$$
\begin{aligned}
& a\left(t_{1}, \ldots, t_{8}\right)=\frac{\theta\left(t_{8} / q t_{6}, t_{6} t_{8}, t_{6} / t_{8} ; p\right)}{\theta\left(t_{8} / t_{7}, t_{7} / q t_{8} ; p\right)} \prod_{k=1}^{5} \theta\left(t_{7} t_{k} / q ; p\right), \\
& \kappa\left(t_{1}, \ldots, t_{8}\right)=\theta\left(t_{7} t_{8} / q ; p\right) \prod_{k=1}^{5} \theta\left(t_{6} t_{k} ; p\right) .
\end{aligned}
$$

Then equation (4.7) can be rewritten as a linear second order $q$-difference equation $\mathcal{D}(\underline{t}) U(\underline{t})=0$, where $U(\underline{t})=V(\underline{t}) / \Gamma_{p, q}\left(t_{7} t_{6}^{ \pm}, t_{8} t_{6}^{ \pm}\right)$. Other linearly independent solutions of this equation can be obtained by replacing $t_{j} \rightarrow p t_{j}$, for some fixed $j=1, \ldots, 5$, or $c \rightarrow p c$, or $x \rightarrow p x$, etc. [19.

It is not difficult to verify the operator identity

$$
\mathcal{D}\left(\underline{t}^{\prime}\right)-\frac{\theta\left(t_{3} / t_{3}^{\prime}, t_{3} / t_{4}^{\prime} ; p\right)}{\theta\left(t_{3} / t_{3}^{\prime \prime}, t_{3} / t_{4}^{\prime \prime} ; p\right)} \mathcal{D}\left(\underline{t}^{\prime \prime}\right)=\frac{t_{3}}{t_{3}^{\prime}} \frac{\theta\left(t_{3}^{\prime} / t_{3}^{\prime \prime}, t_{3}^{\prime} / t_{4}^{\prime \prime} ; p\right)}{\theta\left(t_{3} / t_{3}^{\prime \prime}, t_{3} / t_{4}^{\prime \prime} ; p\right)} \mathcal{D}(\underline{t}),
$$

where in the primed set of parameters $\underline{t}^{\prime}$, the variables $t_{3}$ and $t_{4}$ are replaced by arbitrary $t_{3}^{\prime}$ and $t_{4}^{\prime}$ with the constraint $t_{3} t_{4}=t_{3}^{\prime} t_{4}^{\prime}$ and similarly for the double primed parameters. 
From the elliptic hypergeometric equation $\mathcal{D}(\underline{t}) U(\underline{t})=0$, we find that the ratio

$$
\frac{\mathcal{D}\left(\underline{t}^{\prime}\right) U(\underline{t})}{\theta\left(t_{3} / t_{3}^{\prime}, t_{3} / t_{4}^{\prime} ; p\right)}=\frac{\mathcal{D}\left(\underline{t}^{\prime \prime}\right) U(\underline{t})}{\theta\left(t_{3} / t_{3}^{\prime \prime}, t_{3} / t_{4}^{\prime \prime} ; p\right)}
$$

is independent of the auxiliary parameter $t_{3}^{\prime}$ (or $t_{4}^{\prime}$ ). To find the explicit form of this ratio, we multiply the contiguous relation (4.5) by $\mathcal{A}\left(\underline{t}^{\prime}\right)$ and symmetrize it in the parameters $t_{7}$ and $t_{8}$. After some tedious computations, we obtain

$$
\begin{aligned}
\frac{\mathcal{D}\left(\underline{t}^{\prime}\right) U(\underline{t})}{\theta\left(\frac{t_{3}}{t_{3}^{3}}, \frac{t_{3}}{t_{4}^{3}} ; p\right)}= & t_{4} t_{6} \frac{\theta\left(\frac{t_{3} t_{4} t_{7} t_{8}}{q^{2}}, t_{7} t_{6}, t_{8} t_{6}, \frac{t_{7} t_{8}}{q} ; p\right)}{\theta\left(\frac{t_{3} t_{7}}{q}, \frac{t_{3} t_{8}}{q}, \frac{t_{4} t_{7}}{q}, \frac{t_{4} t_{8}}{q}, \frac{q t_{6}}{t_{5}} ; p\right)} \prod_{j=1, \neq 5,6}^{8} \theta\left(\frac{t_{5} t_{j}}{q} ; p\right) U\left(q^{-1} t_{5}, q t_{6}\right) \\
- & \frac{\theta\left(\frac{q t_{6}}{t_{8}} ; p\right) \prod_{j=1}^{4} \theta\left(t_{6} t_{j} ; p\right)}{\theta\left(\frac{t_{3} t_{8}}{q}, \frac{q}{t_{4} t_{8}} ; p\right)} \\
& \times\left(\frac{\theta\left(t_{6} t_{8}, \frac{t_{7}}{t_{5}}, \frac{t_{5} t_{7}}{q}, \frac{t_{3} t_{4} t_{7} t_{8}}{q^{2}} ; p\right)}{\theta\left(\frac{q t_{6}}{t_{5}}, \frac{t_{3} t_{7}}{q}, \frac{t_{4} t_{7}}{q} ; p\right)}-\frac{\theta\left(\frac{t_{7} t_{8}}{q}, t_{5} t_{6}, \frac{t_{3} t_{4} t_{6} t_{8}}{q} ; p\right)}{\theta\left(t_{3} t_{6}, t_{4} t_{6} ; p\right)}\right) U(\underline{t}) .
\end{aligned}
$$

The elliptic hypergeometric equation follows easily from this relation. Indeed, it suffices to replace $t_{3}^{\prime}$ by $t_{3}^{\prime \prime}$ in (4.10), to subtract the resulting equation from (4.10), and to use the operator identity (4.9).

In the new parameter notation

$$
\varepsilon_{k}=\frac{q}{c t_{k}}, k=1, \ldots, 5, \quad \varepsilon_{6}=c t_{6} p^{4}, \quad \varepsilon_{8}=\frac{c}{t_{6}}, \quad \varepsilon_{7}=\frac{\varepsilon_{8}}{q}
$$

with the balancing condition $\prod_{k=1}^{8} \varepsilon_{k}=p^{2} q^{2}$, the operator $\mathcal{D}$ takes the form

$$
\mathcal{D}_{x}\left(\varepsilon_{1}, \ldots, \varepsilon_{8}\right):=A(x)\left(T_{x, q}-1\right)+A\left(x^{-1}\right)\left(T_{x, q}^{-1}-1\right)+\nu,
$$

where the coefficients

$$
A(x)=\frac{\prod_{k=1}^{8} \theta\left(\varepsilon_{k} x ; p\right)}{\theta\left(x^{2}, q x^{2} ; p\right)}, \quad \nu=\prod_{k=1}^{6} \theta\left(\frac{\varepsilon_{k} \varepsilon_{8}}{q} ; p\right)
$$

obey the explicit $S_{6}$-symmetry in $\varepsilon_{1}, \ldots, \varepsilon_{6}$. Obviously, the equation $\mathcal{D}_{x}(\underline{\varepsilon}) \psi(x ; \underline{\varepsilon})=0$ has a solution $\psi(x ; \underline{\varepsilon})=U\left(t_{1}, \ldots, t_{6}, c x, c x^{-1}\right)$, where

$$
t_{k}=\frac{q}{c \varepsilon_{k}}, k=1, \ldots, 5, \quad t_{6}=\frac{c}{\varepsilon_{8}}, \quad c=\frac{\sqrt{\varepsilon_{6} \varepsilon_{8}}}{p^{2}} .
$$

The operator $\mathcal{D}_{x}(\underline{\varepsilon})$ has the following conjugate with respect to the inner product $\langle\psi, \phi\rangle$ as in (3.3):

$$
\mathcal{D}_{x}^{*}\left(\varepsilon_{1}, \ldots, \varepsilon_{8}\right)=\alpha(x) \mathcal{D}_{x}\left(\varepsilon_{1}, \ldots, \varepsilon_{8}\right) \alpha(x)^{-1}, \quad \alpha(x)=\prod_{k=1}^{8} \Gamma_{p, q}\left(\varepsilon_{k} x^{ \pm}\right),
$$

provided the functions $\psi$ and $\phi$ do not have singularities in the annulus $|q|<|x|<|q|^{-1}$. Therefore, a solution of the equation $\mathcal{D}_{x}^{*}(\varepsilon) \chi(x ; \varepsilon)=0$ is given by the function

$$
\chi(x ; \varepsilon)=\alpha(x) U\left(t_{1}, \ldots, t_{6}, c x^{-1}, c x\right) .
$$

We denote by $\underline{\varepsilon}^{\prime}$ the set of parameters $\underline{\varepsilon}$, where $\varepsilon_{3,4}$ are replaced with $\varepsilon_{3,4}^{\prime}$ such that $\varepsilon_{3} \varepsilon_{4}=\varepsilon_{3}^{\prime} \varepsilon_{4}^{\prime}$ (or, equivalently, $t_{3,4} \rightarrow t_{3,4}^{\prime}, t_{3} t_{4}=t_{3}^{\prime} t_{4}^{\prime}$ ). The obvious identity

$$
0=\left\langle\mathcal{D}_{x}^{*}\left(\underline{\varepsilon}^{\prime}\right) \chi\left(x ; \underline{\varepsilon}^{\prime}\right), \beta(x) \psi(x ; \underline{\varepsilon})\right\rangle=\left\langle\chi\left(x ; \underline{\varepsilon}^{\prime}\right), \mathcal{D}_{x}\left(\underline{\varepsilon}^{\prime}\right) \beta(x) \psi(x ; \underline{\varepsilon})\right\rangle,
$$


where $\beta(x)$ is some function, generates an infinite family of biorthogonality relations. For instance, if $\beta=1$, then the function $\chi\left(x ; \underline{\varepsilon}^{\prime}\right)$ is orthogonal to the function on the righthand side of (4.10) for $\theta\left(\varepsilon_{3}^{\prime} / \varepsilon_{3}, \varepsilon_{4}^{\prime} / \varepsilon_{3}\right) \neq 0$. This complicated biorthogonality is clearly different from (2.12). However, it is plausible that for some particular $\beta(x)$, relation (4.14) yields relation (2.12).

\section{$\S 5$. Relationship with the Sklyanin algebra}

The Sklyanin algebra [14, 15] is generated by four operators $S_{0}, \ldots, S_{3}$ that satisfy the relations

$$
\begin{aligned}
S_{\alpha} S_{\beta}-S_{\beta} S_{\alpha} & =i\left(S_{0} S_{\gamma}+S_{\gamma} S_{0}\right), \\
S_{0} S_{\alpha}-S_{\alpha} S_{0} & =i J_{\beta \gamma}\left(S_{\beta} S_{\gamma}+S_{\gamma} S_{\beta}\right),
\end{aligned}
$$

where the triple $(\alpha, \beta, \gamma)$ represents any cyclic permutation of $(1,2,3)$.

Sklyanin has found the following realization of this algebra in the space of theta functions of even order $2 N$ :

$$
S_{a}=\frac{1}{\theta_{1}(2 u)}\left(s_{a}(u-g) e^{\eta \partial_{u}}-s_{a}(-u-g) e^{-\eta \partial_{u}}\right), \quad g:=\frac{N \eta}{2},
$$

where $e^{ \pm \eta \partial_{u}}$ are the shift operators, $e^{ \pm \eta \partial_{u}} f(u)=f(u \pm \eta)$, and

$$
\begin{aligned}
& s_{0}(u)=\theta_{1}(\eta, 2 u), \quad s_{1}(u)=\theta_{2}(\eta, 2 u)=\theta_{1}(\eta+1 / 2,2 u+1 / 2), \\
& s_{2}(u)=i \theta_{3}(\eta, 2 u)=i e^{\pi i(\tau / 2+\eta+2 u)} \theta_{1}(\eta+1 / 2+\tau / 2,2 u+1 / 2+\tau / 2), \\
& s_{3}(u)=\theta_{4}(\eta, 2 u)=-e^{\pi i(\tau / 2+\eta+2 u)} \theta_{1}(\eta+\tau / 2,2 u+\tau / 2) .
\end{aligned}
$$

The structure constants $J_{\alpha \beta}$ in this case are parametrized as

$$
J_{12}=\frac{\theta_{1}^{2}(\eta) \theta_{4}^{2}(\eta)}{\theta_{2}^{2}(\eta) \theta_{3}^{2}(\eta)}, \quad J_{23}=\frac{\theta_{1}^{2}(\eta) \theta_{2}^{2}(\eta)}{\theta_{3}^{2}(\eta) \theta_{4}^{2}(\eta)}, \quad J_{31}=-\frac{\theta_{1}^{2}(\eta) \theta_{3}^{2}(\eta)}{\theta_{2}^{2}(\eta) \theta_{4}^{2}(\eta)} .
$$

They can be represented in the form $J_{\alpha \beta}=\left(J_{\beta}-J_{\alpha}\right) / J_{\gamma}$, where

$$
J_{1}=\frac{\theta_{2}(2 \eta) \theta_{2}(0)}{\theta_{2}^{2}(\eta)}, \quad J_{2}=\frac{\theta_{3}(2 \eta) \theta_{3}(0)}{\theta_{3}^{2}(\eta)}, \quad J_{3}=\frac{\theta_{4}(2 \eta) \theta_{4}(0)}{\theta_{4}^{2}(\eta)} .
$$

Verification of the defining relations is rather complicated and requires multiple use of the Jacobi identity 12 .

$$
2 \theta_{1}(\vec{b}-B / 2)=\theta_{1}(\vec{b})+\theta_{1}(\vec{b}+1 / 2)+e^{\pi i(\tau+B)}\left(\theta_{1}(\vec{b}+\tau / 2)-\theta_{1}(\vec{b}+1 / 2+\tau / 2)\right),
$$

where $B=b_{1}+b_{2}+b_{3}+b_{4}$ and $\theta_{1}(\vec{b})=\prod_{k=1}^{4} \theta_{1}\left(b_{k}\right)$.

There are two Casimir operators for this algebra. The first is

$$
\begin{aligned}
& K_{0}=\sum_{a=0}^{3} S_{a}^{2} \\
& =\sum_{a=0}^{3}(-1)^{\delta_{a, 2}} \frac{\theta_{a+1}^{2}(\eta)}{\theta_{1}(2 u, 2 u+2 \eta)}\left(\theta_{a+1}(2 u-2 g, 2 u-2 g+2 \eta) e^{2 \eta \partial_{u}}\right. \\
& \left.-\theta_{a+1}(2 u-2 g,-2 u-2 g-2 \eta)\right)+(u \rightarrow-u) \\
& =4 \theta_{1}^{2}(2 g+\eta) .
\end{aligned}
$$

The terms proportional to $e^{ \pm 2 \eta \partial_{u}}$ cancel due to the Jacobi identity with the choice $b_{1}=b_{2}=\eta, b_{3}=2 u-2 g, b_{4}=2 u-2 g+2 \eta$, so that $B=4(u-g+\eta)$ and $\theta_{1}(\vec{b}-B / 2)=0$. The scalar terms combine to $4 \theta_{1}^{2}(2 g+\eta)$ by the same identity with the choice $b_{1}=b_{2}=\eta$, $b_{3}=2 u-2 g, b_{4}=-2 u-2 g-2 \eta$ with $B=-4 g$. 
The second Casimir operator has the form

$$
\begin{aligned}
K_{2} & =\sum_{a=1}^{3} J_{a} S_{a}^{2} \\
& =\sum_{a=1}^{3}(-1)^{\delta_{a, 2}} \frac{\theta_{a+1}^{2}(2 \eta, 0)}{\theta_{1}(2 u, 2 u+2 \eta)}\left(\theta_{a+1}(2 u-2 g, 2 u-2 g+2 \eta) e^{2 \eta \partial_{u}}\right. \\
& \left.-\theta_{a+1}(2 u-2 g,-2 u-2 g-2 \eta)\right)+(u \rightarrow-u) \\
& =4 \theta_{1}(2 g, 2 g+2 \eta) .
\end{aligned}
$$

The terms proportional to $e^{ \pm 2 \eta \partial_{u}}$ cancel due to the Jacobi identity with the choice $b_{1}=0$, $b_{2}=2 \eta, b_{3}=2 u-2 g, b_{4}=-2 u+2 g-2 \eta$, so that $B=0$ and $\theta_{1}(\vec{b}-B / 2)=0$. The scalar terms combine to $4 \theta_{1}(2 g, 2 g+2 \eta)$ by the same identity with the choice $b_{1}=0$, $b_{2}=2 \eta, b_{3}=2 u-2 g, b_{4}=-2 u-2 g-2 \eta$ with $B=-4 g$. We emphasize that all the operator relations described are satisfied for an arbitrary continuous parameter $g$ (i.e., the choice $g=N \eta / 2$ of [15] corresponds to a special finite-dimensional representation in the space of theta functions).

As noticed by Rains [10, 12, a linear combination of the Sklyanin algebra generators $S_{a}$ is equivalent to the $D$-operator. Indeed, this operator is related to the combination

$$
\begin{aligned}
2 \Delta\left(a_{1}, a_{2}, a_{3}, a_{4}\right):= & \frac{\prod_{j=1}^{3} \theta_{1}\left(a_{j}+a_{4}+2 g\right)}{\theta_{1}(\eta)} S_{0}-\frac{\prod_{j=1}^{3} \theta_{1}\left(a_{j}+a_{4}+2 g+\frac{1}{2}\right)}{\theta_{1}\left(\eta+\frac{1}{2}\right)} S_{1} \\
& -i e^{\pi i\left(\frac{\tau}{2}+2 a_{4}+2 g-\eta\right)} \frac{\prod_{j=1}^{3} \theta_{1}\left(a_{j}+a_{4}+2 g+\frac{1+\tau}{2}\right)}{\theta_{1}\left(\eta+\frac{1+\tau}{2}\right)} S_{2} \\
& +e^{\pi i\left(\frac{\tau}{2}+2 a_{4}+2 g-\eta\right)} \frac{\prod_{j=1}^{3} \theta_{1}\left(a_{j}+a_{4}+2 g+\frac{\tau}{2}\right)}{\theta_{1}\left(\eta+\frac{\tau}{2}\right)} S_{3},
\end{aligned}
$$

where we assume that $a_{1}+a_{2}+a_{3}+a_{4}=-4 g$. Substituting explicit expressions for $S_{a}$ and using the Jacobi identity with $b_{1}=a_{1}+a_{4}+2 g, b_{2}=a_{2}+a_{4}+2 g, b_{3}=a_{3}+a_{4}+2 g$, $b_{4}=-2(u-g)$ (so that $\left.B=2 a_{4}+4 g-2 u\right)$, we find

$$
\Delta\left(a_{1}, a_{2}, a_{3}, a_{4}\right)=\frac{\prod_{j=1}^{4} \theta_{1}\left(a_{j}+u\right)}{\theta_{1}(2 u)} e^{\eta \partial_{u}}+\frac{\prod_{j=1}^{4} \theta_{1}\left(a_{j}-u\right)}{\theta_{1}(-2 u)} e^{-\eta \partial_{u}} .
$$

Conversely, any $S_{a}$ can be viewed as a special case of the $\Delta$-operator:

$$
\begin{aligned}
& S_{0}=\chi \theta_{1}(\eta) \Delta\left(-g, \frac{1}{2}-g, \frac{\tau}{2}-g,-\frac{1+\tau}{2}-g\right), \\
& S_{1}=-\chi \theta_{1}\left(\eta+\frac{1}{2}\right) \Delta\left(\frac{1}{4}-g,-\frac{1}{4}-g, \frac{1}{4}+\frac{\tau}{2}-g,-\frac{1}{4}-\frac{\tau}{2}-g\right), \\
& S_{2}=\chi e^{\pi i \eta} \theta_{1}\left(\eta+\frac{1+\tau}{2}\right) \Delta\left(\frac{1+\tau}{4}-g, \frac{1-\tau}{4}-g, \frac{\tau-1}{4}-g,-\frac{1+\tau}{4}-g\right), \\
& S_{3}=\chi e^{\pi i \eta} \theta_{1}\left(\eta+\frac{\tau}{2}\right) \Delta\left(\frac{\tau}{4}-g,-\frac{\tau}{4}-g, \frac{1}{2}+\frac{\tau}{4}-g,-\frac{1}{2}-\frac{\tau}{4}-g\right),
\end{aligned}
$$

where $\chi=i p^{1 / 8} /(p ; p)_{\infty}, p=e^{2 \pi i \tau}$.

In the multiplicative system of notation

$$
(a, b, c, d):=e^{2 \pi i a_{1,2,3,4}}, \quad \rho:=a b c d=e^{-8 \pi i g}, \quad z:=e^{2 \pi i u}, \quad q:=e^{4 \pi i \eta},
$$


the operator $\Delta$ can be rewritten as

$$
\Delta\left(a_{1}, a_{2}, a_{3}, a_{4}\right)=\left(i p^{1 / 8}(p ; p)_{\infty}\right)^{3} e^{4 \pi i g} D(a, b, c, d ; p ; q)
$$

where the operator $D$ coincides with 3.4. In the paper 10 it was indicated that formula (3.11) comprises all commutation relations of the Sklyanin algebra. Thus, the solution of our generalized eigenvalue problem (3.10), fixed in (3.7), determines an infinitedimensional module for the Sklyanin algebra with continuous values of the Casimir operators.

Relation (3.10) describes the action of the $D$-operator on this module $f(z ; w ; a, b ; \rho)$ under a special choice of parameters. To know the action of the Sklyanin algebra generators in general, we need to consider

$$
\begin{aligned}
& D(a, b, c, d ; q ; p) f\left(z ; w ; q^{1 / 2} e, q^{1 / 2} h ; \rho\right) \\
& \quad=\frac{z}{\theta\left(z^{2} ; p\right)}\left(\frac{\theta\left(a z, \ldots, d z, \sqrt{\frac{e h}{\rho}} w^{ \pm} z ; p\right)}{z^{2} \theta(e z, h z ; p)}-\left(z \rightarrow z^{-1}\right)\right) f(z ; w ; e, h ; \rho),
\end{aligned}
$$

where $\rho=a b c d$. We apply now a known identity for theta functions (see Lemma 6.4 in [1]):

$$
\begin{aligned}
z^{-n-1} & \prod_{j=1}^{n} \theta\left(a_{j} z ; p\right) \prod_{k=1}^{n+2} \theta\left(b_{k} z ; p\right)-\left(z \rightarrow z^{-1}\right) \\
= & \frac{(-1)^{n} z \theta\left(z^{-2} ; p\right)}{a_{1} \cdots a_{n}} \sum_{j=1}^{n} \frac{\prod_{k=1}^{n+2} \theta\left(a_{j} b_{k} ; p\right) \prod_{l=1, \neq j}^{n} \theta\left(a_{l} z^{ \pm} ; p\right)}{\prod_{l=1, \neq j}^{n} \theta\left(a_{j} / a_{l} ; p\right)}
\end{aligned}
$$

where $\prod_{j=1}^{n} a_{j} \prod_{k=1}^{n+2} b_{k}=1$. Namely, we choose $n=3, b_{1}=a, \ldots, b_{4}=d, b_{5}=e^{-1}$, $a_{1}=h^{-1}, a_{2,3}=w^{ \pm 1} \sqrt{e h / \rho}$ and find, after some simplifications on the right-hand side of (5.3),

$$
\begin{gathered}
\rho\left(\frac{\theta\left(\sqrt{\frac{e h}{\rho}} a w, \ldots, \sqrt{\frac{e h}{\rho}} d w, \sqrt{\frac{e h}{\rho}} e^{-1} w ; p\right)}{h^{2} \theta\left(h \sqrt{\frac{e h}{\rho}} w, w^{2} ; p\right)} f\left(z ; q^{-1 / 2} w ; q e, h ; \rho\right)+\left(w \rightarrow w^{-1}\right)\right. \\
\left.+\frac{\theta\left(h^{-1} a, \ldots, h^{-1} d, h^{-1} e^{-1} ; p\right)}{\theta\left(h^{-1} \sqrt{\frac{\rho}{e h}} w^{ \pm} ; p\right)} f(z ; w ; q e, q h ; \rho)\right) .
\end{gathered}
$$

Fixing parameters $a_{1,2,3}$ in a different admissible way yields hidden permutational symmetries of this expression. In particular, it is symmetric in $e$ and $h$.

The $D$-operator maps basis vectors to their linear combinations with shifted values of the parameters $e, h$, and $w$. Using relation (2.7) with an appropriate choice of parameters, we can replace $f\left(z ; q^{-1 / 2} w ; q e, h ; \rho\right)$ by the action of some integral operator on the vector $f(z ; x ; q e, q h ; \rho)$ with integration over $x$. This provides a realization of the $D$-operator as an integral operator acting on basis vectors with the parameters qe and $q h$.

In [12, Rosengren proved the Sklyanin conjecture about the reproducing kernel associated with the discrete basis (3.8) and the Sklyanin invariant measure [15. We use a different measure (3.3), i.e., relation (3.14) does not represent a generalization of the similar overlap product for the elliptic $6 j$-symbols in 12 .

\section{$\S 6$. Elliptic analogs of the Faddeev modular Double}

Now we would like to discuss the uniqueness of the basis vectors $f(z ; w ; a, b ; \rho)$. As we saw above, they are fixed up to a constant factor by two generalized eigenvalue problems 
(3.5) and (3.9). The first equation employs linear combinations of the standard Sklyanin algebra generators

$$
S_{a}=i^{\delta_{a, 2}} \frac{\theta_{a+1}(\eta \mid \tau)}{\theta_{1}(2 u \mid \tau)}\left(\theta_{a+1}(2 u-2 g \mid \tau) e^{\eta \partial_{u}}-\theta_{a+1}(-2 u-2 g \mid \tau) e^{-\eta \partial_{u}}\right) .
$$

The second equation requires the introduction of another copy of this algebra, obtained from the first by permutation of $q$ and $p$ (or of $\tau$ and $2 \eta$ ):

$$
\tilde{S}_{a}=i^{\delta_{a, 2}} \frac{\theta_{a+1}\left(\frac{\tau}{2} \mid 2 \eta\right)}{\theta_{1}(2 u \mid 2 \eta)}\left(\theta_{a+1}(2 u-2 g \mid 2 \eta) e^{\frac{\tau}{2} \partial_{u}}-\theta_{a+1}(-2 u-2 g \mid 2 \eta) e^{-\frac{\tau}{2} \partial_{u}}\right) .
$$

For that, we should have $|q|<1$ (i.e., $\operatorname{Im}(\eta)>0$ ). The structure constants $\tilde{J}_{\alpha \beta}$ are also obtained from $J_{\alpha \beta}$ by permutation of $\tau$ and $2 \eta$. Obviously, the $\tilde{S}_{a}$-operators are not well defined in the limit $\operatorname{Im}(\tau) \rightarrow+\infty$; i.e., such a way of fixing representation modules exists only at the elliptic level.

These two sets of operators satisfy the following simple cross-commutation relations:

$$
\begin{array}{lll}
S_{a} \tilde{S}_{b}=\tilde{S}_{b} S_{a}, & a, b \in\{0,3\} & \text { or } \quad a, b \in\{1,2\}, \\
S_{a} \tilde{S}_{b}=-\tilde{S}_{b} S_{a}, & a \in\{0,3\}, b \in\{1,2\} & \text { or } \quad a \in\{1,2\}, b \in\{0,3\} .
\end{array}
$$

The algebra (5.1) has many automorphisms [15]. For real or purely imaginary $\eta$ (i.e., $q^{*}=q^{ \pm 1}$ ), the transformation $S_{a}^{*}=-S_{a}$ is an involution for imaginary $\tau$ (i.e., $p^{*}=p$ ). Since the parameter $\eta$ should lie in the upper half-plane for the $\tilde{S}_{a^{-}}$-operators, such an involution exists for both algebras only for purely imaginary $\eta$. There are specific involutions existing only for our direct product of two Sklyanin algebras. For instance, at $\eta^{*}=-\tau / 2$ (i.e., $q^{*}=p$ ) the conditions $\operatorname{Im}(\tau), \operatorname{Im}(\eta)>0$ are preserved, and we obtain $S_{a}^{*}=-\tilde{S}_{a}$ and $J_{\alpha \beta}^{*}=\tilde{J}_{\alpha \beta}$.

There is a second possibility for fixing solutions of equation (3.5) uniquely. For its description, we use additive notation and substitute $\tau=\omega_{3} / \omega_{2}, 2 \eta=\omega_{1} / \omega_{2}$,

$$
z=e^{2 \pi i \frac{u}{\omega_{2}}}, \quad a, b, c, d=e^{2 \pi i \frac{\alpha, \beta, \gamma, \delta}{\omega_{2}}}, \quad w=e^{2 \pi i \frac{v}{\omega_{2}}}, \quad \rho=e^{2 \pi i \frac{\sigma}{\omega_{2}}}
$$

and $f(z ; w ; a, b ; \rho)=: h(u ; v ; \alpha, \beta ; \sigma)$ in (3.6), which yields

$$
\frac{h\left(u+\frac{\omega_{1}}{2} ; v ; \alpha+\frac{\omega_{1}}{2}, \beta+\frac{\omega_{1}}{2} ; \sigma\right)}{h\left(u-\frac{\omega_{1}}{2} ; v ; \alpha+\frac{\omega_{1}}{2}, \beta+\frac{\omega_{1}}{2} ; \sigma\right)}=z^{4} \frac{\theta\left(a z^{-1}, b z^{-1}, w^{ \pm} z^{-1} \sqrt{c d} ; p\right)}{\theta\left(a z, b z, w^{ \pm} z \sqrt{c d} ; p\right)} .
$$

A solution of this equation valid for $|p|<1$ and $|q| \leq 1$ has the form

$$
h(u ; v ; \alpha, \beta ; \sigma)=\frac{G(\alpha / 2+\beta / 2-\sigma / 2 \pm v \pm u ; \omega)}{G(\alpha \pm u, \beta \pm u ; \omega)},
$$

where $G(u ; \omega)$ is the modified elliptic gamma function. It is defined up to multiplication by an arbitrary function $\varphi(u)$ such that $\varphi\left(u+\omega_{1}\right)=\varphi(u)$. The gamma function $G(u ; \omega)$ is symmetric in $\omega_{1}$ and $\omega_{2}$. It remains a well-defined meromorphic function even for $\omega_{1} / \omega_{2}>0$ (i.e., $|q|=1$ or $\eta$ is real). Demanding that the function (6.2) solve simultaneously equation (6.1) and its partner obtained by permuting $\omega_{1}$ and $\omega_{2}$ for $\omega_{1} / \omega_{2}>0$, we fix $\varphi(u)=$ constant.

In terms of the Sklyanin algebras, now we have two copies of it, with the second algebra obtained from the first by permutation of $\omega_{1}$ and $\omega_{2}$. Such a transformation is equivalent to the changes $\eta \rightarrow 1 / 4 \eta, \tau \rightarrow \tau / 2 \eta$, and $u \rightarrow u / 2 \eta$ in $S_{a}$, which yields

$$
\tilde{S}_{a}=i^{\delta_{a, 2}} \frac{\theta_{a+1}\left(\frac{1}{4 \eta} \mid \frac{\tau}{2 \eta}\right)}{\theta_{1}\left(\frac{u}{\eta} \mid \frac{\tau}{2 \eta}\right)}\left(\theta_{a+1}\left(\frac{u-g}{\eta} \mid \frac{\tau}{2 \eta}\right) e^{\frac{1}{2} \partial_{u}}-\theta_{a+1}\left(\frac{-u-g}{\eta} \mid \frac{\tau}{2 \eta}\right) e^{-\frac{1}{2} \partial_{u}}\right) .
$$


Now we have the following cross-commutation relations for $S_{a}$ and $\tilde{S}_{b}$ :

$$
\begin{array}{lll}
S_{a} \tilde{S}_{b}=\tilde{S}_{b} S_{a}, & a, b \in\{0,1\} & \text { or } \quad a, b \in\{2,3\}, \\
S_{a} \tilde{S}_{b}=-\tilde{S}_{b} S_{a}, & a \in\{0,1\}, b \in\{2,3\} & \text { or } \quad a \in\{2,3\}, b \in\{0,1\} .
\end{array}
$$

Consider the degeneration of these Sklyanin algebra generators in the $\operatorname{limit} \operatorname{Im}\left(\omega_{3} / \omega_{1}\right)$, $\operatorname{Im}\left(\omega_{3} / \omega_{2}\right) \rightarrow+\infty$ (i.e., $r, p \rightarrow 0$ ), which is permissible now. It is not difficult to see that, as $p \rightarrow 0$, the expressions $p^{-1 / 8} S_{0,1}, p^{1 / 8}\left(S_{2}+i S_{3}\right)$, and $p^{-3 / 8}\left(S_{2}-i S_{3}\right)$ have well-defined limits that are finite difference operators; see 4 . Simplifying these operators by shifting $u \rightarrow u+c$ and taking the limit as $\epsilon:=e^{-2 \pi i c} \rightarrow 0$, we obtain three operators $E, F$, and $k$ :

$$
\begin{gathered}
\lim _{\epsilon, p \rightarrow 0} \frac{p^{-1 / 8} i S_{0}}{q^{1 / 4}-q^{-1 / 4}}=k+k^{-1}, \quad \lim _{\epsilon, p \rightarrow 0} \frac{p^{-1 / 8} S_{1}}{q^{1 / 4}+q^{-1 / 4}}=k-k^{-1}, \\
k:=e^{-2 \pi i g+\eta \partial_{u}}, \quad k^{-1}:=e^{2 \pi i g-\eta \partial_{u}}, \\
\lim _{\epsilon, p \rightarrow 0} p^{1 / 8} \frac{S_{2}+i S_{3}}{2 \epsilon}=e^{-2 \pi i u}\left(e^{-\eta \partial_{u}}-e^{\eta \partial_{u}}\right)=:\left(q^{1 / 2}-q^{-1 / 2}\right) F, \\
\lim _{\epsilon, p \rightarrow 0} \epsilon \frac{S_{2}-i S_{3}}{2 p^{3 / 8}}=e^{2 \pi i u}\left(e^{4 \pi i g-\eta \partial_{u}}-e^{-4 \pi i g+\eta \partial_{u}}\right)=:\left(q^{-1 / 2}-q^{1 / 2}\right) E,
\end{gathered}
$$

where $q=e^{4 \pi i \eta}$. The operators $E, F, k$ form the quantum algebra $U_{q}\left(s l_{2}\right)$ with the defining relations

$$
k E=q^{1 / 2} E k, \quad k F=q^{-1 / 2} F k, \quad E F-F E=\frac{k^{2}-k^{-2}}{q^{1 / 2}-q^{-1 / 2}} .
$$

In a similar way, the $\tilde{S}_{a}$-operators degenerate to

$$
\tilde{k}^{ \pm 1}=e^{\mp \frac{\pi i g}{\eta} \pm \frac{1}{2} \partial_{u}}, \quad \tilde{F}=\frac{e^{-\frac{\pi i u}{\eta}}\left(e^{-\frac{1}{2} \partial_{u}}-e^{\frac{1}{2} \partial_{u}}\right)}{e^{\frac{\pi i}{2 \eta}}-e^{-\frac{\pi i}{2 \eta}}}, \quad \tilde{E}=\frac{e^{\frac{\pi i u}{\eta}}\left(e^{\frac{2 \pi i g}{\eta}-\frac{1}{2} \partial_{u}}-e^{-\frac{2 \pi i g}{\eta}+\frac{1}{2} \partial_{u}}\right)}{e^{-\frac{\pi i}{2 \eta}}-e^{\frac{\pi i}{2 \eta}}},
$$

forming the $U_{\tilde{q}^{-1}}\left(s l_{2}\right)$-algebra

$$
\tilde{k} \tilde{E}=\tilde{q}^{-1 / 2} \tilde{E} \tilde{k}, \quad \tilde{k} \tilde{F}=\tilde{q}^{1 / 2} \tilde{F} \tilde{k}, \quad \tilde{E} \tilde{F}-\tilde{F} \tilde{E}=\frac{\tilde{k}^{2}-\tilde{k}^{-2}}{\tilde{q}^{-1 / 2}-\tilde{q}^{1 / 2}},
$$

with $\tilde{q}=e^{-\pi i / \eta}$. Thus, we obtain the Faddeev modular double [3].

In [3, it was demanded that generators of the copies of quantum algebras commute with each other. This requirement is satisfied by the explicit realization of the modular double used in [6]. In our case, not all the generators of two algebras commute with each other:

$$
k \tilde{F}=-\tilde{F} k, \quad k \tilde{E}=-\tilde{E} k, \quad \tilde{k} F=-F \tilde{k}, \quad \tilde{k} E=-E \tilde{k},
$$

which seems to contradict this demand. However, introducing the operator $K=k^{2}$ and rewriting the defining relations of the $U_{q}\left(s l_{2}\right)$-algebra as

$$
K E=q E K, \quad K F=q^{-1} F K, \quad E F-F E=\frac{K-K^{-1}}{q^{1 / 2}-q^{-1 / 2}},
$$

we obtain the desired commutativity of the generators $E, F, K$ with $\tilde{E}, \tilde{F}, \tilde{K}$, as in 6 ] (note that $q$ and $\tilde{q}$ of the paper [6] are equal to our parameters $q^{1 / 2}$ and $\tilde{q}^{-1 / 2}$, respectively). At the elliptic level, such a quadratic change is not natural; one has to work with the operators $S_{a}$ and $\tilde{S}_{a}$, some of which anticommute.

For $|q|=1$, the algebra $U_{q}\left(s l_{2}\right)$ has the involution $E^{*}=-E, F^{*}=-F, K^{*}=K$. As noticed by Faddeev $\left[3\right.$, for $q^{*}=\tilde{q}$ the modular double has another involution permuting the algebras in the pair: $E^{*}=-\tilde{E}, F^{*}=-\tilde{F}, K^{*}=\tilde{K}$. For our direct product of the Sklyanin algebras, in the first case we have $S_{a}^{*}=-S_{a}$ and $\tilde{S}_{a}^{*}=-\tilde{S}_{a}$, provided $J_{\alpha \beta}^{*}=J_{\alpha \beta}$ 
and $\tilde{J}_{\alpha \beta}^{*}=\tilde{J}_{\alpha \beta}$ (or equivalently, $|q|=1$ and $p^{*}=p$ ). An elliptic analog of the Faddeev involution has the form $S_{a}^{*}=-\tilde{S}_{a}$, and it exists under the condition $J_{\alpha \beta}^{*}=\tilde{J}_{\alpha \beta}$. These constraints for the structure constants are satisfied if $|2 \eta|=1$ (i.e., $\eta^{*}=1 / 4 \eta$ or $q^{*}=\tilde{q}$ ) and $\tau^{*}=-\tau / 2 \eta$ (i.e., $\left.p^{*}=r\right)$. Equivalently, $2 \eta=e^{i \phi}, \phi \in(-\pi, \pi)$, and $\tau=i \rho e^{i \phi / 2}$, $\rho>0$.

Summarizing our consideration, we conclude that the second way of fixing functional freedom in the definition of basis vectors provides an elliptic generalization of the Faddeev modular doubling principle. The first way of fixing the function space has no evident trigonometric limit, i.e., at the elliptic level there are two different "modular doubles".

\section{$\S 7$. Bethe eigenfunctions and Relationship with the Heun EQuation}

As shown by Sklyanin, all operators $S_{a}$ preserve the space of even theta functions of order $2 N$ if

$$
g=\frac{N \eta}{2}
$$

Therefore, the operator $\Delta\left(a_{1}, \ldots, a_{4}\right)$ obeys the same property (its multivariate generalization to the root system $B C_{n}$, proposed by Rains [9], obeys a similar property). Thus, one can try to diagonalize it in the space of such functions:

$$
\Delta\left(a_{1}, \ldots, a_{4}\right) \psi(u)=E \psi(u), \quad \psi(u)=\prod_{m=1}^{N} \theta_{1}\left(u \pm u_{m}\right),
$$

$\psi(-u)=\psi(u)$. This equation has the following explicit form:

$$
\begin{aligned}
\frac{\prod_{k=1}^{4} \theta_{1}\left(a_{k}+u\right)}{\theta_{1}(2 u)} \prod_{m=1}^{N} \theta_{1}\left(u \pm u_{m}+\eta\right) & +\frac{\prod_{k=1}^{4} \theta_{1}\left(a_{k}-u\right)}{\theta_{1}(-2 u)} \prod_{m=1}^{N} \theta_{1}\left(u \pm u_{m}-\eta\right) \\
& =E \prod_{m=1}^{N} \theta_{1}\left(u \pm u_{m}\right), \quad \sum_{k=1}^{4} a_{k}=-2 N \eta .
\end{aligned}
$$

If we divide this relation by $\psi(u)$, then on the left-hand side we obtain two elliptic functions of $u$ of order $2 N+4$, and a constant on the right-hand side. In order to verify this elliptic functions identity, it suffices therefore to check its validity for $2 N+5$ different values of $u$ in the fundamental parallelogram of periods for some particular choice of the spectral parameter $E$.

First, it is easily verified for $u=0,1 / 2, \tau / 2,(1+\tau) / 2$, when $\theta_{1}(2 u)=0$. Second, the validity of (7.1) at $u= \pm u_{j}$ yields $N$ Bethe Anzatz equations for the roots $u_{1}, \ldots, u_{N}$ :

$$
\prod_{k=1}^{4} \frac{\theta_{1}\left(a_{k}+u_{m}\right)}{\theta_{1}\left(a_{k}-u_{m}\right)}=\prod_{n=1}^{N} \frac{\theta_{1}\left(u_{m} \pm u_{n}-\eta\right)}{\theta_{1}\left(u_{m} \pm u_{n}+\eta\right)}, \quad m=1, \ldots, N .
$$

Actually, it suffices to satisfy any $N-1$ equations among (7.2), because elliptic functions cannot have only one simple pole in the fundamental region. As a $(2 N+5)$ th value of $u$ for which (7.1) is valid, we take $u=a_{l}$ for some $l$, which fixes the parameter $E$ as

$$
E=\frac{\prod_{k=1}^{4} \theta_{1}\left(a_{k}+a_{l}\right)}{\theta_{1}\left(2 a_{l}\right)} \prod_{n=1}^{N} \frac{\theta_{1}\left(a_{l} \pm u_{n}+\eta\right)}{\theta_{1}\left(a_{l} \pm u_{n}\right)} .
$$

Apparently, this expression should not depend on the choice of $l=1,2,3,4$, which is rather easy to check for $N=0$.

In a similar way, a more general Bethe Anzatz $\psi(u)=e^{\gamma u} \prod_{k=1}^{2 N} \theta_{1}\left(u-u_{k}\right)$ can be analyzed for some constant $\gamma$ determined by the eigenvalue problem $\Delta \psi=E \psi$. There are solutions different from (7.1). For instance, if the operator $\Delta$ is reduced to $S_{0}$, then 
for $N=2 n$ the roots $u_{k}$ can be chosen in such a way that $\psi(u)=e^{\gamma u} \prod_{k=1}^{n} \theta_{1}\left(2\left(u-u_{k}\right)\right)$ and the Bethe equations have solutions with a nontrivial parameter $\gamma$ (see the review 13 . and the references therein). All these special eigenfunctions are expected to be related to some finite difference operators commuting with $\Delta$ and forming some algebraic relations with it.

Now we consider the limit as $\eta \rightarrow 0$, corresponding to the transition from finite differences to differentiations. For that, we perform a gauge transformation:

$$
\begin{aligned}
\tilde{\Delta}\left(a_{1}, \ldots, a_{4}\right) & =\chi \rho^{-1}(u) \Delta\left(a_{1}, \ldots, a_{4}\right) \rho(u) \\
& =\chi \frac{\rho(u+\eta)}{\rho(u)} \frac{\theta_{1}(\vec{a}+u)}{\theta_{1}(2 u)} e^{\eta \partial_{u}}-\chi \frac{\rho(u-\eta)}{\rho(u)} \frac{\theta_{1}(\vec{a}-u)}{\theta_{1}(2 u)} e^{-\eta \partial_{u}},
\end{aligned}
$$

where $\chi$ is some constant to be specified later. Choosing $\rho(u)$ as a solution of the equation

we find

$$
\rho(u+\eta)=\frac{1}{\chi} \frac{\theta_{1}(2 u)}{\theta_{1}(\vec{a}+u)} \rho(u),
$$

The potential

$$
\tilde{\Delta}\left(a_{1}, \ldots, a_{4}\right)=e^{\eta \partial_{u}}-\chi^{2} \frac{\theta_{1}(u-\vec{a}, u+\vec{a}-\eta)}{\theta_{1}(2 u, 2 u-2 \eta)} e^{-\eta \partial_{u}} .
$$

$$
\frac{\theta_{1}(u-\vec{a}, u+\vec{a}-\eta)}{\theta_{1}(2 u, 2 u-2 \eta)}
$$

is an elliptic function of $u$ of the eighth order with periods 1 and $\tau$. We choose

$$
a_{1}=\alpha_{0} \eta, \quad a_{2}=\frac{1}{2}+\alpha_{1} \eta, \quad a_{3}=\frac{\tau}{2}+\alpha_{2} \eta, \quad a_{4}=-\frac{1+\tau}{2}+\alpha_{3} \eta,
$$

and consider the limit as $\eta \rightarrow 0$. Fixing

$$
\chi=\frac{i p^{1 / 8} e^{\pi i\left(\alpha_{2}-\alpha_{3}\right) \eta}}{(p ; p)_{\infty}^{3}}
$$

after some computations we find $\tilde{\Delta}\left(a_{1}, \ldots, a_{4}\right)=2-\eta^{2} L+O\left(\eta^{3}\right)$, where

$$
L=-\frac{d^{2}}{d u^{2}}+\sum_{i=0}^{3} \alpha_{i}\left(\alpha_{i}-1\right) \mathcal{P}\left(u+\frac{\omega_{i}}{2}\right)
$$

and $\omega_{0}=0, \omega_{1}=1, \omega_{2}=\tau, \omega_{3}=\omega_{1}+\omega_{2}$, and the function $\mathcal{P}(u)=-d^{2} \log \theta_{1}(u) / d u^{2}$ coincides up to an additive constant with the Weierstrass function with periods 1 and $\tau$.

The eigenvalue problem for the $L$-operator is known to lead to the Heun equation with the general set of parameters. Thus, the equation determining the spectrum of the $\Delta$-operator represents a finite difference analog of the Heun equation. For this reason, we call $\Delta\left(a_{1}, \ldots, a_{4}\right)$ (or $D(a, b, c, d ; p ; q)$ ) the difference Heun operator.

Since the Heun equation is related to the one-particle Hamiltonian of the Inozemtsev model [5] (an elliptic Calogero-Sutherland type model for the $B C_{n}$-root system). Therefore, is it natural to expect that the $D$-operator has a similar relationship with relativistic quantum multiparticle systems. Indeed, this is true for the model proposed by van Diejen [2] and investigated in detail by Komori and Hikami [7. In the presence of the balancing condition $\prod_{j=1}^{8} \varepsilon_{j}=p^{2} q^{2}$, the corresponding one-particle Hamiltonian takes the form

$$
H=A(x)\left(T_{x, q}-1\right)+A\left(x^{-1}\right)\left(T_{x, q}^{-1}-1\right), \quad A(x)=\frac{\prod_{m=1}^{8} \theta\left(\varepsilon_{m} x ; p\right)}{\theta\left(x^{2}, q x^{2} ; p\right)} .
$$

Imposing the additional constraints

$$
\varepsilon_{1}=q^{1 / 2}, \quad \varepsilon_{2}=-q^{1 / 2}, \quad \varepsilon_{3}=(p q)^{1 / 2}, \quad \varepsilon_{4}=-(p q)^{1 / 2}
$$

and replacing $\varepsilon_{5}$ with $p \varepsilon_{5}$, we find $\prod_{j=5}^{8} \varepsilon_{j}=1$ and

$$
H=-\varepsilon_{5}^{-1}\left(D\left(\varepsilon_{5}, \ldots, \varepsilon_{8} ; p ; q^{2}\right)-\varepsilon_{5}^{-1} \theta\left(\varepsilon_{5} \varepsilon_{6}, \varepsilon_{5} \varepsilon_{7}, \varepsilon_{5} \varepsilon_{8} ; p\right)\right) .
$$


A more detailed consideration of the relationship between this model and the $D$-operator lies beyond the scope of the present paper. We see that the difference Heun operator is a rather universal object: it emerges in the Calogero-Sutherland type models, and it generates the Sklyanin algebra and a family of biorthogonal functions related to the elliptic analog of the Gauss hypergeometric function.

The standard eigenvalue problem for the Hamiltonian (7.4) with a special choice of parameters and for a special eigenvalue coincides with the elliptic hypergeometric equation [20. Therefore, a particular eigenfunction of the $D$-operator is related to the $V$-function. To find it, we substitute $t_{1,2}= \pm q^{1 / 2} c^{-1}, t_{3,4}= \pm(p q)^{1 / 2} c^{-1}$ in (4.8) and find that, under these conditions,

$$
\begin{aligned}
& a\left(t_{1}, \ldots, t_{7}, t_{8}\right)+a\left(t_{1}, \ldots, t_{8}, t_{7}\right)=\kappa\left(t_{1}, \ldots, t_{8}\right), \\
& \mathcal{D}\left(t_{1}, \ldots, t_{8}\right)=-\frac{t_{6}}{c} D\left(c t_{6}, \frac{c}{t_{6}}, \frac{c}{q t_{6}}, \frac{p q}{c t_{5}} ; p ; q^{2}\right) .
\end{aligned}
$$

This means that the function

$$
\psi_{1}(x)=\frac{V\left(\frac{q^{1 / 2}}{c},-\frac{q^{1 / 2}}{c}, \frac{(p q)^{1 / 2}}{c},-\frac{(p q)^{1 / 2}}{c}, t_{5}, t_{6}, c x^{-1}, c x\right)}{\Gamma_{p, q}\left(c x^{ \pm} t_{6}^{ \pm}\right)},
$$

where $t_{5} t_{6}=p c^{2}$, is a zero mode of the resulting $D$-operator. However, this zero mode is easily computable from scratch,

$$
\psi_{2}(x)=\frac{\Gamma_{p, q^{2}}\left(\frac{q^{2} t_{6}}{c} x^{ \pm}, \frac{c t_{5}}{p} x^{ \pm}\right)}{\Gamma_{p, q^{2}}\left(q c t_{6} x^{ \pm}, \frac{q c}{t_{6}} x^{ \pm}\right)}
$$

The meromorphic solutions of the equation $D \psi(x)=0$ are defined up to multiplication by an arbitrary function satisfying $\varphi\left(q^{2} x\right)=\varphi(x)$; i.e., the ratio $\psi_{1}(x) / \psi_{2}(x)$ is an elliptic function of $x$.

The Heun equation is a general second order differential equation with four regular singular points. A description of its general solution in closed form is an important open mathematical problem. From our consideration we see that the solutions of the generalized eigenvalue problem for the difference Heun operator determine a basis of functions, the connection problem for which is solved by the $R$-function (2.8).

The modular parameter $\tau$ can be viewed as a time variable for the Hamilton dynamics generated by the classical analog of the operator (7.3). Then the equations of motion are known to be equivalent to the Painlevé-VI equation [8. In a similar way, we can take the classical analog of the $D$-operator and interpret it as a nonstationary Hamitonian with the time variable $\tau$. For a special dependence of the parameters $a_{j}$ on $\tau$, the corresponding equations of motion would define some particular $q$-deformation of the Painlevé-VI function.

The biorthogonality (2.10) is easily generalizable to a similar relation for the entire hierarchy of integrals (2.1). Therefore, it looks possible to generalize the construction described in this paper to $I^{(m)}$ with $m>1$. Also, it is natural to conjecture that there exist analogs of the basis vectors $f(z ; w ; a, b ; \rho)$ for the root system $B C_{n}$, which can be built with the help of the generalized eigenvalue problem for the Rains operator [9]. Their determination is a challenging problem for the future.

After completion of this work, L. D. Faddeev communicated to the author that an anticommutativity related to the $k, \tilde{k}$-operators (6.3) was also noticed in the paper by L. D. Faddeev and A. Yu. Volkov, Discrete evolution for the zero-modes of the quantum Liouville model, arXiv:0803.0230. 


\section{ACKNOWLEDGMENTS}

The author is indebted to V. B. Priezzhev for stimulating discussions and to A. N. Kirillov, E. M. Rains, and H. Rosengren for useful remarks on this paper. Relations (2.7) and (3.10) were presented for the first time in a slightly different form at the conference Riemann-Hilbert Problems, Integrability, and Asymptotics (September 20-25, 2005, SISSA, Trieste). The organizers of that conference are thanked for their warm hospitality. This work was supported in part by the Russian Foundation for Basic Research (RFBR), grant no. 06-01-00191.

\section{REFERENCES}

[1] G. E. Andrews, R. Askey, and R. Roy, Special functions, Encyclopedia Math. Appl., vol. 71, Cambridge Univ. Press, Cambridge, 1999. MR.1688958 (2000g:33001)

[2] J. F. van Diejen, Integrability of difference Calogero-Moser systems, J. Math. Phys. 35 (1994), 2983-3004. MR1275485 (95j:58066)

[3] L. D. Faddeev, Modular double of a quantum group, Conference Moshé Flato 1999, Vol. I (Dijon), Math. Phys. Stud., vol. 21, Kluwer, Dordrecht, 2000, pp. 149-156. MR1805888 (2002a:81126)

[4] A. S. Gorsky and A. V. Zabrodin, Degenerations of Sklyanin algebra and Askey-Wilson polynomials, J. Phys. A 26 (1993), L635-L639. MR.1239346 (94j:81108)

[5] V. I. Inozemtsev, Lax representation with spectral parameter on a torus for integrable particle systems, Lett. Math. Phys. 17 (1989), 11-17. MR0990577(90j:58058)

[6] S. Kharchev, D. Lebedev, and M. Semenov-Tian-Shansky, Unitary representations of $U_{q}(s l(2, \mathbb{R}))$, the modular double and the multiparticle q-deformed Toda chains, Comm. Math. Phys. 225 (2002), 573-609. MR 1888874 (2003b:17019)

[7] Y. Komori and K. Hikami, Quantum integrability of the generalized elliptic Ruijsenaars models, J. Phys. A 30 (1997), 4341-4364. MR1478324 (99g:81242)

[8] Yu. I. Manin, Sixth Painlevé equation, universal elliptic curve, and mirror of $P^{2}$, Geometry of Differential Equations, Amer. Math. Soc. Transl. (2), vol. 186, Amer. Math. Soc., Providence, RI, 1998, pp. 131-151. MR1732409 (2001b:14086)

[9] E. M. Rains, Transformations of elliptic hypergeometric integrals, Ann. of Math. (2) (to appear).

[10] — $B C_{n}$-symmetric Abelian functions, Duke Math. J. 135 (2006), 99-180. MR.2259924 (2008e:33043)

[11] H. Rosengren, An elementary approach to $6 j$-symbols (classical, quantum, rational, trigonometric, and elliptic), Ramanujan J. 13 (2007), 131-166. MR.2281159 (2008c:33018)

[12] _ Sklyanin invariant integration, Int. Math. Res. Not. 2004, no. 60, 3207-3232. MR2096219 (2005m:33024)

[13] S. N. M. Ruijsenaars, Special functions associated with Calogero-Moser type quantum systems, Integrable Systems: from Classical to Quantum (Montréal, QC, 1999), CRM Proc. Lecture Notes, vol. 26, Amer. Math. Soc., Providence, RI, 2000, pp. 189-226. MR1791891 (2001k:33039)

[14] E. K. Sklyanin, Some algebraic structures connected with the Yang-Baxter equation, Funktsional. Anal. i Prilozhen. 16 (1982), no. 4, 27-34; English transl., Funct. Anal. Appl. 16 (1982), no. 4. 263-270 (1983). MR0684124(84c:82004)

[15] _ Some algebraic structures connected with the Yang-Baxter equation. Representations of a quantum algebra, Funktsional. Anal. i Prilozhen. 17 (1983), no. 4, 34-48; English transl., Funct. Anal. Appl. 17 (1983), no. 4, 273-284. MR0725414 (85k:82011)

[16] V. P. Spiridonov, On the elliptic beta function, Uspekhi Mat. Nauk 56 (2001), no. 1, 181-182; English transl., Russian Math. Surveys 56 (2001), no. 1, 185-186. MR1846786 (2003b:33032)

[17] _ Theta hypergeometric integrals, Algebra i Analiz 15 (2003), no. 6, 161-215; English transl., St. Petersburg Math. J. 15 (2004), no. 6, 929-967. MR2044635 (2005m:33030)

[18] _ Bailey's tree for integrals, Teoret. Mat. Fiz. 139 (2004), no. 1, 104-111; English transl., Theoret. and Math. Phys. 139 (2004), no. 1, 536-541. MR2076912 (2005f:33030)

[19] —_ Elliptic hypergeometric functions, Doctor Diss., LTF OIYaI, 2004, 218 pp. (Russian) (see also math.CA/0704.3099)

[20] - Elliptic hypergeometric functions and models of Calogero-Sutherland type, Teoret. Mat. Fiz. 150 (2007), no. 2, 311-324; English transl., Theoret. and Math. Phys. 150 (2007), no. 2, 266-277. MR2325931 (2008d:81102)

[21] T. Takebe, Bethe ansatz for higher spin eight-vertex models, J. Phys. A 28 (1995), 6675-6706. MR $1381138(97 \mathrm{c}: 82023 \mathrm{a})$ 
[22] L. A. Takhtadzhyan and L. D. Faddeev, The quantum method for the inverse problem and the $X Y Z$ Heisenberg model, Uspekhi Mat. Nauk 34 (1979), no. 5, 13-63; English transl. in Russian Math. Surveys 34 (1979), no. 5. MR0562799 (81d:82066)

[23] A. S. Zhedanov, Biorthogonal rational functions and the generalized eigenvalue problem, J. Approx. Theory 101 (1999), 303-329. MR1726460(2000i:33014)

Bogoliubov Laboratory of Theoretical Physics, JinR, Dubna, 141980 Moscow Region, RusSIA

E-mail address: spiridon@theor.jinr.ru

Received 17/DEC/2007

Translated by THE AUTHOR 